\title{
Consensus Reaching in Multiple Attribute Group Decision Making: A Multi-Stage Optimization Feedback Mechanism with Individual Bounded Confidences
}

\author{
Quanbo Zha, Yucheng Dong, Francisco Chiclana, Enrique Herrera-Viedma
}

\begin{abstract}
Existing consensus models focus on improving the group consensus level, but ignore whether a higher group consensus level means higher mutual acceptance of decision makers. In the field of opinion dynamics, the bounded confidence model asserts that the decision makers will accept the preferences of others within a neighborhood of theirs with width a certain confidence level. Inspired by this research methodology, this paper develops a consensus model to address the acceptance issue based on individual bounded confidences. Specifically, a bounded confidence-based consensus measure is designed to measure the level of group mutual acceptance, and a multi-stage optimization feedback mechanism based on individual bounded confidences is proposed to maximize the group mutual acceptance and minimize the amount of preference adjustment. A numerical example and a simulation analysis are included to illustrate the use of the model and to justify its effectiveness, respectively.
\end{abstract}

Index Terms-Group decision making; Consensus; Bounded confidence; Multi-Stage Optimization

\section{INTRODUCTION}

$\mathrm{T}_{\mathrm{n}}^{\mathrm{o}}$ obtain a solution with agreement in group decision making (GDM), it is necessary to include a consensus

We would like to acknowledge the financial support of the grants (Nos. 71871149 and 72001031) from NSF of China, the grant (No. 2020M673146) from China Postdoctoral Science Foundation, the grant (No. 2020YJ0043) from Sichuan Provincial Science and Technology Planning Project, and the project (PID2019-103880RB-I00/AEI/10.13039/501100011033) from Spanish State Research Agency. (Corresponding author: Quanbo Zha)

Q.B. Zha is with the School of Management Science and Real Estate, Chongqing University, Chongqing 400045, China (e-mail: qbzha@cqu.edu.cn).

Y.C. Dong is with the Center for Network Big Data and Decision-Making, Business School, Sichuan University, Chengdu 610065, China (e-mail: ycdong@scu.edu.cn).

F. Chiclana is with the Institute of Artificial Intelligence, School of Computer Science and Informatics, De Montfort University, LE1 9BH Leicester, UK, and also with the Andalusian Research Institute in Data Science and Computational Intelligence, University of Granada, 18010 Granada, Spain (e-mail: chiclana@dmu.ac.uk).

E. Herrera-Viedma is with the Andalusian Research Institute in Data Science and Computational Intelligence, University of Granada, 18010 Granada, Spain, and also with the Department of Electrical and Computer Engineering, Faculty of Engineering, King Abdulaziz University, Jeddah 21589, Saudi Arabia (e-mail: viedma@decsai.ugr.es). process within the resolution procedure [1]-[5], with tools to support the enhancement of consensus level among the group via an iterative process of preference adjustment. Consensus measurement and feedback mechanism are two key phases normally included in consensus process. The first phase is to measure and quantify the consensus level among the group, while the second phase is often embodied in the form of consensus rules that generate preference adjustment recommendations to increase the group consensus level.

Currently, measurement of consensus is mainly based on the use of a distance function in two main methods: (1) Measurement based on the distances between the individual decision makers' preferences and the collective preference. Spillman et al.'s research on consensus within the fuzzy sets framework [6] being one of the earliest approaches to develop a distance based consensus measure; other notable examples of developing distance based consensus measures are Herrera et al.'s linguistic preferences consensus measures [7] and Ben-Arieh and Chen's order based consensus measure and mean based consensus measure [8]. (2) Measurement based on pairwise distances between decision makers' preferences. Kacprzyk and Fedrizzi et al. [9] developed the consensus measurement to capture the similarity between decision makers' preferences from the perspective of "soft"; Herrera et al. [10] proposed consensus measurement for linguistic preferences based on the concept of coincidence of linguistic values; while Chen et al. [11] investigated consensus measurement based on deviation and overlap degrees in GDM with uncertain linguistic terms.

Feedback mechanism is mainly expressed in the form of consensus rules and includes two types: (1) The first type of rule is known as the identification rule and direction rule (IR-DR) [12]-[14], where IR identifies the decision makers in unacceptable states of consensus levels who are advised by DR to adjust their preferences in the appropriate direction. Herrera-Viedma et al. [13] studied the feedback mechanism in the form of IR-DR for pursuing higher consensus level in a multigranular linguistic preference relation framework; Zhang et al. [15] used IR-DR within the uncertain 2-tuple linguistic preference relations framework; while Dong et al. [16] and Wu et al. [17] proposed trust relationships consensus models with IR-DR. (2) The second type of rule is known as the optimization-based consensus rule [18]-[20], because it aims to 
minimize the adjustments/costs. Zhang et al. [53] stuided minimum cost consensus models and their economic significance; while Ben-Arieh and Easton [22] investigated the minimum cost issue with multiple attributes; Zhang et al. [21] developed the minimum adjustments in a 2-rank context; while $\mathrm{Wu}$ et al. [23] studied this issue in GDM with trust relationships.

In the existing consensus models, the willingness to accept the feedback recommendations has been studied in the form of bounded confidence in recent years. In opinion dynamics, the bounded confidence model defines this psychological behavior, that is, decision maker will only accept the preferences within their bounded confidence [24]-[26]. Zhang et al. [27] developed a two-stage consensus model with bounded confidence; Liang et al. [28] studied this issue in minimum adjustments consensus model with time constraints; Zha et al. [29] proposed a bounded confidence learning mechanism in GDM; Zhang et al. [56] studied GDM with bounded confidence within linguistic preference context; Zhang et al. [57] considered leadership and bounded confidence in social network GDM; while Dong et al. [54] proposed a hybrid GDM framework using bounded confidence to obtain stable opinions. However, there are still some limitations in the existing studies:

(1) In the existing consensus models, the measurement of consensus are based on a distance function as described above, which may not ensure that decision makers will accept the final decision result. Indeed, even if the preferences of two decision makers are similar, there is still a certain distance between their preferences that may be larger than their respective bounded confidence levels leading to their disagreement. On the other hand, even if the preferences of two decision makers are not similar, the two may agree with each other because of their larger psychological bottom line.

(2) In a consensus process, the feedback mechanism improves the similarities of the preferences of decision makers without considering the improvement of decision makers' mutual acceptance. However, the mutual acceptance among decision makers is one of the characteristics describing consensus, since it affects decision makers' satisfaction on the final decision results. In other words, the existing feedback mechanisms can effectively enhance the similarity of group preferences but not the mutual acceptance among decision makers.

To overcome the above limitations, this paper proposes a bounded confidence based consensus model with multi-stage optimization feedback mechanism (MOFMCM) for multiple attribute GDM (MAGDM) problems, aiming at helping promote the level of group mutual acceptance improvement. The specific contributions of this paper are:

(1) A consensus measurement methodology to quantify the level of group mutual acceptance based on the bounded confidence model in opinion dynamics;

(2) A multi-stage optimization feedback mechanism based on bounded confidence: (i) maximizing the level of mutual acceptance, (ii) minimizing the preference adjustments after maximizing acceptance, (iii) maximizing the similarity of the group when mutual acceptance cannot be improved, and (iv) minimizing preference adjustments after maximizing similarity;

(3) Simulation and comparison analysis methodology to justify the effectiveness of MOFMCM in improving group mutual acceptance.

The rest of this article is arranged as follows. In Section II, the general consensus process framework, the minimum adjustment consensus model, and the bounded confidence model are presented. The resolution process for the MAGDM problem with bounded confidence is described in Section III. Section IV illustrates the use of the MOFMCM and analyzes its effectiveness in increasing mutual acceptance. Finally, conclusions are drawn in Section V.

\section{PRELIMINARIES}

This section reviews briefly the main architecture of a general consensus process in MAGDM (Section II-A), the minimum adjustment consensus model (Section II-B), and the bounded confidence model (Section II-C).

\section{A. A General Consensus Process in MAGDM}

The main objective of an MAGDM problem is to arrive at a consensus-based solution from multiple alternatives on multiple attributes. The typical elements of an MAGDM problem are:

(1) A group of decision makers $D=\left\{d_{1}, d_{2}, \cdots, d_{r}\right\}(r \geq 2)$ with associated weights $\omega=\left\{\omega_{1}, \omega_{2}, \cdots, \omega_{r}\right\}$ subject to constraints: $\omega_{k} \geq 0(k=1,2, \cdots, r) ; \sum_{k=1}^{r} \omega_{k}=1$.

(2) A finite set of alternatives $X=\left\{x_{1}, x_{2}, \cdots, x_{m}\right\}(m \geq 2)$, which are the potential solutions of the MAGDM problem;

(3) A set of attributes $A=\left\{a_{1}, a_{2}, \cdots, a_{n}\right\}(n \geq 2)$ with associated weights $w=\left\{w_{1}, w_{2}, \cdots, w_{n}\right\}(n \geq 2)$ subject to the constraints: $w_{j} \geq 0(j=1,2, \cdots, n) ; \sum_{j=1}^{n} w_{j}=1$.

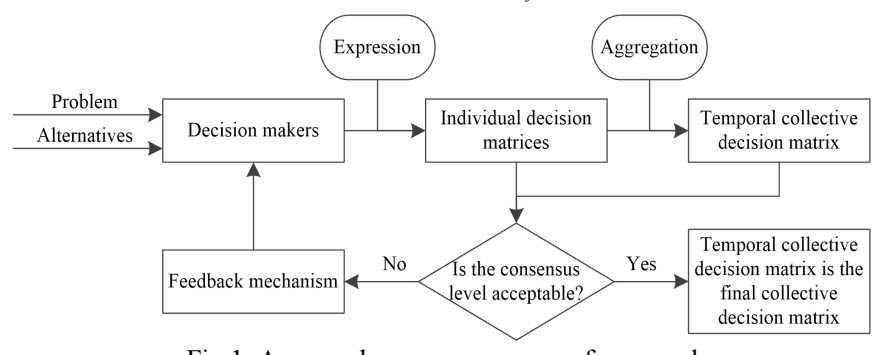

Fig.1. A general consensus process framework

A consensus process is an iterative process of preference adjustment with the purpose of improving the group consensus level. Fig. 1 shows its most general basic process, which consists of two fundamental phases:

(1) Consensus measurement: This phase quantifies the group consensus level by the first method as described before [14], [31], [39], [48]. Let $V^{k}=\left(v_{i j}^{k}\right)_{m \times n}$ be the multiple attribute decision matrix expressed by decision maker $d_{k} \in D$, where $v_{i j}^{k} \in[0,1]$ denotes the evaluation value for the alternative $x_{i} \in X$ with respect to the attribute $a_{j} \in A$. Without loss of generality, this paper uses the weighted average (WA) operator [32] (different aggregation operators are applicable) to compute the collective decision matrix $V^{c}=\left(v_{i j}^{c}\right)_{m \times n}$ :

$$
v_{i j}^{c}=f_{\omega}\left(v_{i j}^{1}, v_{i j}^{2}, \cdots, v_{i j}^{r}\right)=\sum_{k=1}^{r} \omega_{k} v_{i j}^{k}
$$


Applying the Manhattan distance, the individual consensus level of $d_{k} \in D$ is:

$$
C L\left(d_{k}\right)=1-\frac{\sum_{i=1}^{m} \sum_{j=1}^{n}\left|v_{i j}^{k}-v_{i j}^{c}\right|}{m n}
$$

The weighted average of the individual consensus levels is the group consensus level:

$$
C L=\sum_{k=1}^{r} \omega_{k} \operatorname{cl}\left(d_{k}\right)
$$

The larger $C L \in[0,1]$, the higher the group consensus level. Let $\mu \in[0,1]$ be 'the group consensus threshold' (closer to 1 ). If $C L<\mu$, then the next consensus fundamental phase is activated because of the unacceptable or unsatisfactory current group consensus level. Otherwise, the current collective decision matrix is considered as the final one.

(2) Feedback mechanism: This phase provides feedback recommendations for increasing the group consensus level. IR and DR are two classical consensus rules employed in this phase [12], [13]. IR is employed for identifying the decision maker(s) with unsatisfactory consensus level(s). DR provides feedback recommendations of preference adjustment, $\bar{V}^{k}=\left(\bar{v}_{i j}^{k}\right)_{m \times n}$, to the identified decision makers $d_{k} \in D$ with the goal of increasing their consensus levels:

$$
\bar{v}_{i j}^{k} \in\left[\min \left(v_{i j}^{k}, v_{i j}^{c}\right), \max \left(v_{i j}^{k}, v_{i j}^{c}\right)\right]
$$

When an satisfactory group consensus level is achieved, via the iterative application of the above two consensus phases, a selection process is activated to derive a final ranking of the alternatives $\left(x_{i}\right)$ based on their corresponding dominance values $\left(Q_{i}\right)$ [33], [34]:

$$
Q_{i}=W A_{w}\left(v_{i 1}^{c}, v_{i 2}^{c}, \cdots, v_{i n}^{c}\right)=\sum_{j=1}^{n} w_{j} v_{i j}^{c}
$$

\section{B. The Minimum Adjustment Consensus Model}

As described in section II-A, the general consensus model uses the IR-DR to promote the group to achieve a consensus. However, IR and DR based feedback mechanism may cause a large amount of loss of the original preference information. To improve the efficiency of group consensus, Dong et al. [18] proposed an original minimum adjustment consensus model. To simplify the illustration here, this model is illustrated in the form of a multiple attribute decision matrix as the preference of decision maker.

This model retains the original preferences of decision makers as much as possible. If $V^{k}=\left(v_{i j}^{k}\right)_{m \times n}$ and $\bar{V}^{k}=\left(\bar{v}_{i j}^{k}\right)_{m \times n}$ are the original and adjusted multiple attribute decision matrix mentioned in Section II-A, then the objective function to optimize is the distance between $V^{k}$ and $\bar{V}^{k}, D\left(V^{k}, \bar{V}^{k}\right)$, i.e.

$$
\min \sum_{k=1}^{r} D\left(V^{k}, \bar{V}^{k}\right)
$$

Meanwhile, the individual consensus level should be at an acceptable level, i.e.

$$
c l^{\prime}\left(d_{k}\right) \leq \alpha
$$

where $c l^{\prime}\left(d_{k}\right)=\sum_{i=1}^{m} \sum_{j=1}^{n}\left|\bar{v}_{i j}^{k}-\bar{v}_{i j}^{c}\right| / m n ; \bar{V}^{c}$ is the collective decision matrix obtained from the adjusted multiple attribute decision matrices via the aggregation function $f_{\pi}\left(\bar{V}_{1}, \cdots, \bar{V}_{r}\right)$; and $\alpha \in[0,1]$ (closer to 0 ) is the consensus threshold. Thus, the optimal adjusted preferences, $\left\{\bar{V}_{1}, \cdots, \bar{V}_{r}\right\}$, which are the feedback recommendations, are obtained by solving the below optimization model:

$$
\begin{aligned}
& \min \sum_{k=1}^{r} D\left(V^{k}, \bar{V}^{k}\right) \\
& \begin{cases}\text { s.t. } & \sum_{i=1}^{m} \sum_{j=1}^{n}\left|\bar{v}_{i j}^{k}-\bar{v}_{i j}^{c}\right| / m n \leq \alpha \quad k=1, \cdots, r \\
& \bar{V}^{c}=f_{\pi}\left(\bar{V}_{1}, \cdots, \bar{V}_{r}\right)\end{cases}
\end{aligned}
$$

So far, the minimum adjustment consensus model has been widely studied in the following scenarios [46]: (1) using linguistic preferences (Dong et al. [18]; Wu et al. [40]); (2) using preference relations (Zhang et al. [41]; Wu et al. [42]; Zhang et al. [43]); (3) using heterogeneous preference representation structures (Chen et al. [44]; Zhang et al. [45]); (4) in MAGDM (Zha et al. [30]; Yu et al. [47]); (5) using a multi-stage optimization strategy (Zhang et al. [19]; Wu et al. [50]); (6) in classification-based GDM (Zhang et al. [21]; Chen et al. [49]); (7) in social network GDM (Wu et al. [23]; Cheng et al. [51]); (8) in large-scale GDM (Zha et al. [30]; Xiao et al. [52]); and (9) in opinion dynamic GDM (Liang et al. [28]; Chen et al. [39]; Dong et al. [53]).

\section{Bounded Confidence Model}

In opinion dynamics, Hegselmann-Krause (HK) model [35] and Deffuant-Weisbuch (DW) model [36], [37], are two widespread bounded confidence models. Both models study individual willingness of accepting opinions, and argue that decision makers will only be influenced by the recommendations similar to their own opinions. Specifically, the bounded confidence of a decision maker is the critical value to judge whether a recommendation is acceptable to the decision maker.

Let $V^{R}=\left(v_{i j}^{R}\right)_{m \times n}$ be the feedback recommendation for adjusting decision matrix $V^{k}=\left(v_{i j}^{k}\right)_{m \times n}$ of decision maker $d_{k}$ in a consensus process. Let $B=\left\{b_{1}^{k}, b_{2}^{k}, \cdots, b_{n}^{k}\right\}$ be the bounded confidence set of decision maker $d_{k}$ associated with the attributes $A=\left\{a_{1}, a_{2}, \cdots, a_{n}\right\}(n \geq 2)$. According to the bounded confidence model, decision maker $d_{k}$ will accept the feedback recommendation $v_{i j}^{R}$ when the distance between $v_{i j}^{R}$ and $v_{i j}^{k}$, $D\left(v_{i j}^{R}, v_{i j}^{k}\right)$, is less than or equal to $b_{j}^{k}$, i.e.

$$
\left|v_{i j}^{R}-v_{i j}^{k}\right| \leq b_{j}^{k}
$$

In both HK and DW models, the bounded confidence influences the convergence time and the distribution of final opinions [35]-[37]. The larger the bounded confidence value, the smaller the number of opinion clusters and the larger the opinion cluster size. When the bounded confidence takes a sufficiently large value, an opinion cluster is formed between the decision makers, that is, consensus is reached. In GDM 
problems, consensus model has begun to pay attention to the bounded confidence model [27], [29], [30], [54]. Zha et al. [29] provided a learning algorithm to find out the unknown bounded confidence. However, in most existing studies, the research paradigm of GDM with bounded confidence assumes that the bounded confidences are given or known. Therefore, in this paper, we follow the research line of the GDM with bounded confidence and assume that the bounded confidences are known.

\section{CONSENSUS Model With Multi-STAGE Optimization FEEDBACK MECHANISM}

This section proposes a consensus model with individual bounded confidences based on a multi-stage optimization feedback mechanism.

\section{A. Framework of the MOFMCM Model}

In a consensus process under bounded confidence context, decision makers will accept or reject feedback recommendations according to their own bounded confidences [29], [30]. However, the existing bounded confidence based consensus models ignore the mutual acceptance among decision makers. Even if the distance-based consensus is at a high level, decision makers may be unsatisfied with the final solution since other decision makers' preferences may be unacceptable for them based on their own bounded confidences. Therefore, it is meaningful to measure the consensus level taking into account the mutual acceptance of decision makers. Thus, this section develops a consensus-based solution for MAGDM problem with individual bounded confidences, where the consensus level and the multi-stage optimization feedback mechanism are both designed based on the level of mutual acceptance among decision makers.

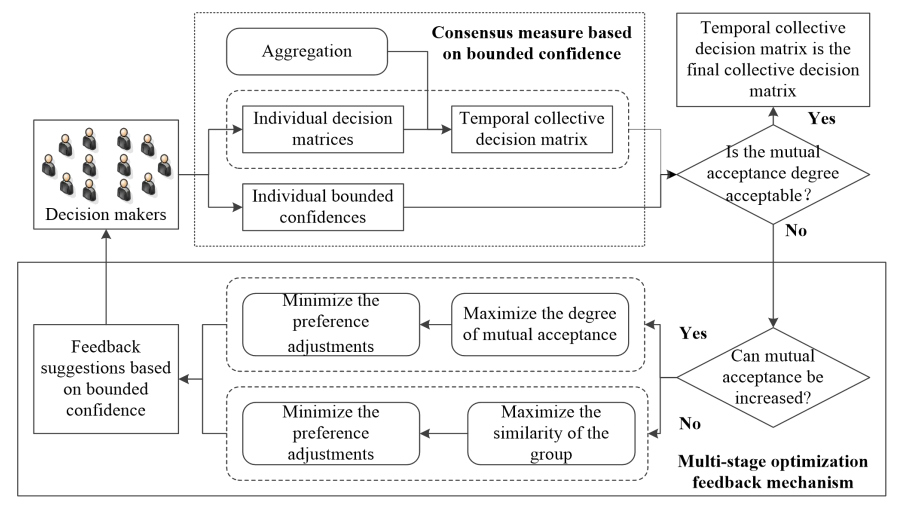

Fig. 2. Framework of the MOFMCM model

The MOFMCM model framework shown in Fig. 2 includes two key phases: (1) Consensus measure based on bounded confidence, and (2) multi-stage optimization feedback mechanism. In the first phase group consensus level is measured based on the mutual acceptance level among the group. The second phase is divided into two parts: (1) A two-stage optimization based on mutual acceptance to improve the mutual acceptance between decision makers, and (2) a two-stage optimization based on similarity which is activated when the first part cannot be improved, to improve the similarity between decision makers.

\section{B. Consensus Measure based on Bounded Confidence}

As mentioned in the introduction, two main methods for measuring group consensus level aim to narrow the preference distance between decision makers. However, they ignored whether decision makers agreed to the current level of consensus at each round. In other words, the decision makers' preferences may be relatively similar but with a context of mutual rejection among them; on the contrary, the decision makers' preferences may not be similar but within a context of mutual acceptance of them. This is illustrated with the following example.

Example 1: Let $B^{1}=\{0.1,0.05,0.6\}$ and $B^{2}=\{0.14,0.04,0.56\}$ be the bounded confidences of decision makers $d_{1}$ and $d_{2}$, respectively. And their decision matrices are as follows:

$$
V^{1}=\left(\begin{array}{lll}
0.25 & 0.63 & 0.90 \\
0.70 & 0.53 & 0.35 \\
0.81 & 0.39 & 0.15
\end{array}\right), \quad V^{2}=\left(\begin{array}{lll}
0.67 & 0.56 & 0.58 \\
0.24 & 0.46 & 0.80 \\
0.41 & 0.45 & 0.63
\end{array}\right) .
$$

Based on Eq. (6), we draw the following observations:

(1) Decision makers $d_{1}$ and $d_{2}$ are far away from each other with respect to their evaluations with respect to attribute $a_{1}$, and each one of them consider the other's opinions are unacceptable as suggestions.

(2) Decision makers $d_{1}$ and $d_{2}$ have close evaluations with respect to attribute $a_{2}$, but each one of them consider the other's opinions unacceptable as suggestions.

(3) Decision makers $d_{1}$ and $d_{2}$ are still far apart in their evaluations with respect to attribute $a_{3}$, but they are mutually acceptable.

In what follows, the group consensus level is derived from the measurements of the levels of mutual acceptance among decision makers based on individual bounded confidences.

Let $B=\left\{b_{1}^{k}, b_{2}^{k}, \cdots, b_{n}^{k}\right\}$ be the bounded confidence set of decision maker $d_{k}$ associated with the set of attributes $A=\left\{a_{1}, a_{2}, \cdots, a_{n}\right\} \quad(n \geq 2) \quad$. Let $\quad A D^{l k}=\left(a d_{i j}^{l k}\right)_{m \times n}$ $(k, l=1,2, \cdots, r ; k \neq l)$ be a $0-1$ matrix to represent the decision maker $d_{l}$ acceptance of decision maker $d_{k}: a d_{i j}^{l k}=1$ denotes decision maker $d_{l}$ accepts decision maker $d_{k}$ evaluation value for alternative $x_{i} \in X$ with respect to the attribute $a_{j} \in A$; otherwise, $a d_{i j}^{l k}=0$. According to the bounded confidence model, we have

$$
a d_{i j}^{l k}= \begin{cases}1 & \text { if }\left|v_{i j}^{k}-v_{i j}^{l}\right| \leq b_{j}^{l} \\ 0 & \text { if }\left|v_{i j}^{k}-v_{i j}^{l}\right|>b_{j}^{l}\end{cases}
$$

The following acceptance levels are defined:

(1) The acceptance level from decision maker $d_{l}$ to decision maker $d_{k}$ :

$$
A L^{l k}=\frac{\sum_{i=1}^{m} \sum_{j=1}^{n} a d_{i j}^{l k}}{m n}
$$

(2) The group's acceptance level of decision maker $d_{k}$ : 


$$
A L\left(d_{k}\right)=\frac{\sum_{l=1, l \neq k}^{r} A L^{l k}}{r-1}
$$

(3) The group mutual acceptance level:

$$
A L=\frac{\sum_{k=1}^{r} A L\left(d_{k}\right)}{r}
$$

Obviously, $A L \in[0,1]$. And larger $A L$ values indicate higher mutual acceptance levels. It can be seen from Eqs. (10)-(13) that under the same preference, the greater the boundary trust, the greater the degree of mutual acceptance. In this paper, we use the group mutual acceptance level to measure consensus instead of the traditional method described by Eq. (3). For notation simplicity, we still use $\mu \in[0,1]$ to denote the mutual acceptance threshold. If $A L<\mu$, then group mutual acceptance is unsatisfactory, and the feedback phase is activated. Otherwise, Eq. (5) will be used to obtain the dominance values of alternatives and the final solution to the MAGDM problem.

\section{Multi-stage Optimization Feedback Mechanism}

Existing feedback mechanisms aim to increase the distance based group consensus level by improving the similarity between decision makers without considering the decision makers' recognition of the consensus level improvement. This section proposes a multi-stage optimization feedback mechanism based on bounded confidence, which includes two parts: (1) a two-stage optimization based on mutual acceptance, and (2) a two-stage optimization based on similarity.

As shown in Example 1, the mutual acceptance of attribute $a_{1}$ between the two decision makers cannot be improved. This situation may happen for the entire group. When the two-stage optimization based on mutual acceptance cannot improve the group mutual acceptance, the MOFMCM model will activate the two-stage optimization based on similarity to enhance the similarity of the decision makers' decision matrices and lay the foundation for increasing the mutual acceptance among decision makers in next round.

(1) A two-stage optimization based on mutual acceptance. This consists of two consecutive stages: Stage (i) to maximize the mutual acceptance, and Stage (ii) to minimize the preference adjustments based on Stage (i).

Stage (i) aims to maximize the group mutual acceptance, i.e., the feedback decision matrices $\bar{V}^{k}$ and $\bar{V}^{l}$ should maximize the number of 1 elements in $A D^{l k}=\left(a d_{i j}^{l k}\right)_{m \times n}$ $(k, l=1,2, \cdots, r ; k \neq l)$ :

$$
\begin{gathered}
\max A L \quad\left(\text { or } \max \frac{1}{\operatorname{mnr}(r-1)} \sum_{l=1, l \neq k}^{r} \sum_{k=1}^{r} \sum_{i=1}^{m} \sum_{j=1}^{n} a d_{i j}^{l k}\right) \\
a d_{i j}^{l k}= \begin{cases}1 & \text { if }\left|\bar{v}_{i j}^{k}-\bar{v}_{i j}^{l}\right| \leq b_{j}^{l} \\
0 & \text { if }\left|\bar{v}_{i j}^{k}-\bar{v}_{i j}^{l}\right|>b_{j}^{l}\end{cases}
\end{gathered}
$$

In order to make the feedback decision matrix $\bar{V}^{k}$ within the acceptable range of the decision maker $d_{k}$, the feedback decision matrix $\bar{V}^{k}$ and the decision matrix $V^{k}$ need to meet the following constraint:

$$
\left|\bar{v}_{i j}^{k}-v_{i j}^{k}\right| \leq b_{j}^{k}
$$

Based on Eqs. (14)-(16), the mutual acceptance maximization model becomes:

$$
\begin{gathered}
\min -\frac{1}{m n r(r-1)} \sum_{l=1, l \neq k}^{r} \sum_{k=1}^{r} \sum_{i=1}^{m} \sum_{j=1}^{n} a d_{i j}^{l k} \\
\left\{\begin{array}{cl}
\text { s.t. }\left|\bar{v}_{i j}^{k}-v_{i j}^{k}\right| \leq b_{j}^{k} & i=1,2, \cdots, m ; j=1,2, \cdots, n ; \\
a d_{i j}^{l k}= \begin{cases}1 \text { if }\left|\bar{v}_{i j}^{k}-\bar{v}_{i j}^{l}\right| \leq b_{j}^{l} & i=1,2, \cdots, m ; j=1,2, \cdots, n ; \\
0 \text { if }\left|\bar{v}_{i j}^{k}-\bar{v}_{i j}^{l}\right|>b_{j}^{l} & l, k=1,2, \cdots, r ; l \neq k\end{cases} \\
0 \leq \bar{v}_{i j}^{k} \leq 1 & i=1,2, \cdots, m ; j=1,2, \cdots, n ; \\
& k=1,2, \cdots, r
\end{array}\right.
\end{gathered}
$$

The optimal solution for $A L$ denoted by $A L^{*}$, is the solution of model (17), which stands the highest level of mutual acceptance that the group can reach. Nevertheless, multiple solutions for $\bar{V}^{k}(k=1,2, \cdots, r)$ may exist in model (17). Therefore, we further optimize the optimal solution of the feedback decision matrices through Stage (ii).

Stage (ii) aims to minimize the group preference adjustments on the basis of the optimal solution $A L^{*}$, i.e., the distances between the feedback decision matrix $\bar{V}^{k}$ and the decision matrix $V^{k}$ needs to be minimized:

$$
\min \frac{1}{r m n} \sum_{k=1}^{r} \sum_{i=1}^{m} \sum_{j=1}^{n}\left|\bar{v}_{i j}^{k}-v_{i j}^{k}\right|
$$

where $a d_{i j}^{l k}$ is computed by Eq. (15), while constraint (16) being still valid at Stage (ii). To achieve the highest level of mutual acceptance, the following constraint is to be met:

$$
\frac{1}{m n r(r-1)} \sum_{l=1, l \neq k}^{r} \sum_{k=1}^{r} \sum_{i=1}^{m} \sum_{j=1}^{n} a d_{i j}^{l k}=A L^{*}
$$

According to Eqs. (15)-(19), the preference adjustment minimization model becomes:

$$
\begin{aligned}
& \min \frac{1}{r m n} \sum_{k=1}^{r} \sum_{i=1}^{m} \sum_{j=1}^{n}\left|\bar{v}_{i j}^{k}-v_{i j}^{k}\right| \\
& \left\{\begin{array}{lll}
\text { s.t. }\left|\bar{v}_{i j}^{k}-v_{i j}^{k}\right| \leq b_{j}^{k} & i=1,2, \cdots, m ; j=1,2, \cdots, n ; \\
a d_{i j}^{l k}= \begin{cases}1 \text { if }\left|\bar{v}_{i j}^{k}-\bar{v}_{i j}^{l}\right| \leq b_{j}^{l} & k=1,2, \cdots, r \\
0 \text { if }\left|\bar{v}_{i j}^{k}-\bar{v}_{i j}^{l}\right|>b_{j}^{l} & i=1,2, \cdots, m ; j=1,2, \cdots, n ; \\
\frac{1}{m n r(r-1)} \sum_{l=1, l, \neq k}^{r} \sum_{k=1}^{r} \sum_{i=1}^{m} \sum_{j=1}^{n} a d_{i j}^{l k}=A L^{*} & \\
0 \leq \bar{v}_{i j}^{k} \leq 1 & i=1, k=1,2, \cdots, r ; l \neq k\end{cases} \\
& k=1,2, \cdots, m, r
\end{array}\right.
\end{aligned}
$$

The optimal value for $\bar{V}^{k}(k=1,2, \cdots, r)$ denoted $\bar{V}^{k *}$, is the solution of model (20). Model (20) has a closed bounded non-empty feasible region. Therefore, it can be known from the Weierstrass theorem that the optimal solution(s) of model (20) exists. However, model (20) may still have multiple optimal solutions. For this kind of issues, it can be further optimized based on these optimal solutions. For example, Chandran et al. [58] developed a two-stage approach to pursue a unique optimal solution; Dong and Herrera-Viedma [59] also agreed with this solution approach. Specifically, let $\bar{V}^{*}$ be the optimal solution set of model (20), and then the unique optimal solution can be obtain based on model $\left(20^{\prime}\right)$ : 


$$
\min _{\bar{V}^{k} * \bar{V}^{*}} \max _{k} D\left(\bar{V}^{k *}, V^{k}\right)
$$

Model $\left(20^{\prime}\right)$ further minimizes the maximal distances between $\bar{V}^{k} *$ and $V^{k}$, and can be similarly solved. This paper does not focus on the uniqueness issue, so we do not discuss this issue in detail here.

To improve the group mutual acceptance, it is recommended that the adjusted decision matrix value $v_{i j}^{k^{\prime}}$ of decision maker $d_{k}$ follows the rule below:

$$
v_{i j}^{k^{\prime}} \in\left[\min \left(v_{i j}^{k}, \bar{v}_{i j}^{k}\right), \max \left(v_{i j}^{k}, \bar{v}_{i j}^{k} *\right)\right]
$$

(2) A two-stage optimization based on similarity. This part also includes two stages of optimization: Stage (i) to maximize the similarity of the group, and stage (ii) to minimize preference adjustments.

Stage (i) aims to increase the proximity of the decision makers' decision matrices by minimizing the distance between the individual feedback decision matrices $\bar{V}^{k}(k=1,2, \cdots, r)$ and their corresponding collective decision matrix $\bar{V}^{c}$,

$$
\begin{array}{r}
D\left(\bar{V}^{k}, \bar{V}^{c}\right)=\sum_{i=1}^{m} \sum_{j=1}^{n}\left|\bar{v}_{i j}^{k}-\bar{v}_{i j}^{c}\right| / m \cdot n, \text { i.e., } \\
\min \sum_{k=1}^{r} D\left(\bar{V}^{k}, \bar{V}^{c}\right)
\end{array}
$$

Constraint (16) is used here to ensure that the adjusted decision matrix is within the bounded confidence range of the decision maker. Based on Eq. (1), we have $\bar{v}_{i j}^{c}=\sum_{k=1}^{r} \omega_{k} \bar{v}_{i j}^{k}$. Then, the model similarity maximization model becomes:

$$
\begin{aligned}
& \min \sum_{k=1}^{r} D\left(\bar{V}^{k}, \bar{V}^{c}\right) \\
& \left\{\begin{array}{lll}
\text { s.t. } & \mid \bar{v}_{i j}^{k}-v_{i j}^{k} \leq b_{j}^{k} & i=1,2, \cdots, m ; j=1,2, \cdots, n \\
& \bar{v}_{i j}^{c}=\sum_{k=1}^{r} \omega_{k} \bar{v}_{i j}^{k} & i=1,2, \cdots, m ; j=1,2, \cdots, n \\
0 \leq \bar{v}_{i j}^{k} \leq 1 & i=1,2, \cdots, m ; j=1,2, \cdots, n
\end{array}\right.
\end{aligned}
$$

The optimal values for $\bar{V}^{k}$ and $\bar{V}^{c}$, denoted as $\bar{V}^{k * *}$ and $\bar{V}^{c * *}$, are the solution for model (23). Based on Eqs. (2) and (3), the highest level of group similarity (i.e., group consensus level) $C L^{*}$ is:

$$
C L^{*}=1-\frac{\sum_{k=1}^{r} \sum_{i=1}^{m} \sum_{j=1}^{n}\left|\bar{v}_{i j}^{k} * *-\bar{v}_{i j}^{c} * *\right|}{r m n}
$$

However, model (23) may multiple solutions for $\bar{V}^{k}$ $(k=1,2, \cdots, r)$. Therefore, Stage (ii) further minimizes the preference adjustments based on the highest group similarity to optimize the optimal solution for $\bar{V}^{k}(k=1,2, \cdots, r)$. To achieve this, Eq. (19) is employed with the group similarity subject to the following constraint:

$$
1-\frac{1}{r m n} \sum_{k=1}^{r} \sum_{i=1}^{m} \sum_{j=1}^{n}\left|\bar{v}_{i j}^{k}-\bar{v}_{i j}^{c}\right|=C L^{*}
$$

According to Eqs. (16) and (25), the preference adjustment minimization model based on the highest group similarity become:

$$
\begin{array}{ll}
\min \frac{1}{r m n} \sum_{k=1}^{r} \sum_{i=1}^{m} \sum_{j=1}^{n}\left|\bar{v}_{i j}^{k}-v_{i j}^{k}\right| & \\
\text { s.t. } \quad\left|\bar{v}_{i j}^{k}-v_{i j}^{k}\right| \leq b_{j}^{k} & i=1,2, \cdots, m ; j=1,2, \cdots, n ; \\
\bar{v}_{i j}^{c}=\sum_{k=1}^{r} \omega_{k} \bar{v}_{i j}^{k} & k=1,2, \cdots, r \\
1-\frac{1}{r m n} \sum_{k=1}^{r} \sum_{i=1}^{m} \sum_{j=1}^{n}\left|\bar{v}_{i j}^{k}-\bar{v}_{i j}^{c}\right|=C L^{*} & \\
0 \leq \bar{v}_{i j}^{k} \leq 1 & k=1,2, \cdots, \cdots, r \\
& i=1,2, \cdots, m ; j=1,2, \cdots, n ; \\
& k=1,2, \cdots, r
\end{array}
$$

The optimal value for $\bar{V}^{k}(k=1,2, \cdots, r)$, namely $\bar{V}^{k * *^{\prime}}$, is the solution of model (23). To improve the group similarity, it is recommended that the adjusted decision matrix value $v_{i j}^{k^{\prime}}$ of decision maker $d_{k}$ follows the rule below:

$$
v_{i j}^{k^{\prime}} \in\left[\min \left(v_{i j}^{k}, \bar{v}_{i j}^{k * * \prime}\right), \max \left(v_{i j}^{k}, \bar{v}_{i j}^{k * * \prime}\right)\right]
$$

\section{Mixed 0-1 Linear Programming Associated with the MOFMCM Model}

To facilitate solving model (17), this is transformed into a mixed 0-1 linear programming model. Lemma 1 is the theoretical basis for equivalent transformation.

Lemma 1: Let $M$ be a large enough number. When $\bar{v}_{i j}^{k}$ and $\bar{v}_{i j}^{l}$ satisfy constraint (28), we have

$$
\begin{aligned}
& a d_{i j}^{l k}=\left\{\begin{array}{ll}
1 & \text { if }\left|\bar{v}_{i j}^{k}-\bar{v}_{i j}^{l}\right| \leq b_{j}^{l} \\
0 & \text { if }\left|\bar{v}_{i j}^{k}-\bar{v}_{i j}^{l}\right|>b_{j}^{l}
\end{array} .\right. \\
& \begin{cases}\bar{v}_{i j}^{k}-\bar{v}_{i j}^{l} \geq\left(y_{i j}^{l k}-1\right) M & i=1,2, \cdots, m ; j=1,2, \cdots, n ; \\
& l, k=1,2, \cdots, r ; l \neq k \\
\bar{v}_{i j}^{k}-\bar{v}_{i j}^{l}<y_{i j}^{l k} M & i=1,2, \cdots, m ; j=1,2, \cdots, n ; \\
& l, k=1,2, \cdots, r ; l \neq k \\
\bar{v}_{i j}^{k}-\bar{v}_{i j}^{l} \leq b_{j}^{l}+\left(2-y_{i j}^{l k}-a d_{i j}^{l k}\right) M & i=1,2, \cdots, m ; j=1,2, \cdots, n ; \\
& l, k=1,2, \cdots, r ; l \neq k \\
\bar{v}_{i j}^{k}-\bar{v}_{i j}^{l}>b_{j}^{l}-\left(1-y_{i j}^{l k}+a d_{i j}^{l k}\right) M & i=1,2, \cdots, m ; j=1,2, \cdots, n ; \\
& l, k=1,2, \cdots, r ; l \neq k \\
-\bar{v}_{i j}^{k}+\bar{v}_{i j}^{l} \leq b_{j}^{l}+\left(y_{i j}^{l k}+1-a d_{i j}^{l k}\right) M & i=1,2, \cdots, m ; j=1,2, \cdots, n ; \\
& l, k=1,2, \cdots, r ; l \neq k \\
-\bar{v}_{i j}^{k}+\bar{v}_{i j}^{l}>b_{j}^{l}-\left(y_{i j}^{l k}+a d_{i j}^{l k}\right) M & i=1,2, \cdots, m ; j=1,2, \cdots, n ; \\
y_{i j}^{l k}, r d_{i j}^{l k} \in\{0,1\} & l, k=1,2, \cdots, r ; l \neq k \\
& i=1,2, \cdots, m ; j=1,2, \cdots, n ; \\
& l, k=1,2, \cdots, r ; l \neq k\end{cases}
\end{aligned}
$$

Proof: From $\bar{v}_{i j}^{k}-\bar{v}_{i j}^{l} \geq\left(1-y_{j i}^{l k}\right) M$ and $\bar{v}_{i j}^{k}-\bar{v}_{i j}^{l}<y_{j i}^{l k} M$, we obtain that: (1) $y_{i j}^{l k}=1$ implies $\bar{v}_{i j}^{k}-\bar{v}_{i j}^{l} \geq 0$; (2) while $y_{i j}^{l k}=0$ implies $\bar{v}_{i j}^{k}-\bar{v}_{i j}^{l}<0$.

Furthermore, from $\bar{v}_{i j}^{k}-\bar{v}_{i j}^{l} \leq b_{j}^{l}+\left(2-y_{j i}^{l k}-a d_{i j}^{l k}\right) M \quad$ and $\bar{v}_{i j}^{k}-\bar{v}_{i j}^{l}>b_{j}^{l}-\left(1-y_{j i}^{l k}+a d_{i j}^{l k}\right) M$, we have: (3) When $y_{i j}^{l k}=1$, from (1) we have $\bar{v}_{i j}^{k}-\bar{v}_{i j}^{l} \geq 0$. Then, $a d_{i j}^{l k}=1$ can obtain $0 \leq \bar{v}_{i j}^{k}-\bar{v}_{i j}^{l} \leq b_{j}^{l}$ and $\bar{v}_{i j}^{k}-\bar{v}_{i j}^{l} \geq 0>b_{j}^{l}-M$; while $a d_{i j}^{l k}=0$ implies $0 \leq \bar{v}_{i j}^{k}-\bar{v}_{i j}^{l} \leq M$ and $\bar{v}_{i j}^{k}-\bar{v}_{i j}^{l}>b_{j}^{l} \geq 0$; (4) When 
$y_{i j}^{l k}=0, \bar{v}_{i j}^{k}-\bar{v}_{i j}^{l}<0$ can be obtained from (2). Then, $a d_{i j}^{l k}=1$ implies $\bar{v}_{i j}^{k}-\bar{v}_{i j}^{l}<0 \leq b_{j}^{l}+M \quad$ and $0>\bar{v}_{i j}^{k}-\bar{v}_{i j}^{l}>b_{j}^{l}-2 M$; while $a d_{i j}^{l k}=0$ can obtain $\bar{v}_{i j}^{k}-\bar{v}_{i j}^{l}<0 \leq b_{j}^{l}+2 M \quad$ and $0>\bar{v}_{i j}^{k}-\bar{v}_{i j}^{l}>b_{j}^{l}-M$.

Then, it is $a d_{i j}^{l k}=\left\{\begin{array}{ll}1 & \text { if } 0 \leq \bar{v}_{i j}^{k}-\bar{v}_{i j}^{l} \leq b_{j}^{l} \\ 0 & \text { if } \bar{v}_{i j}^{k}-\bar{v}_{i j}^{l}>b_{j}^{l} \geq 0\end{array}\right.$.

Subsequently, from $-\bar{v}_{i j}^{k}+\bar{v}_{i j}^{l} \leq b_{j}^{l}+\left(1+y_{j i}^{l k}-a d_{i j}^{l k}\right) M$ and $-\bar{v}_{i j}^{k}+\bar{v}_{i j}^{l}>b_{j}^{l}-\left(y_{j i}^{l k}+a d_{i j}^{l k}\right) M$, we have that: (5) When $y_{i j}^{l k}=1$, based on (1), $\quad \bar{v}_{i j}^{k}-\bar{v}_{i j}^{l} \geq 0$. Furthermore, $a d_{i j}^{l k}=1$ implies $-\bar{v}_{i j}^{k}+\bar{v}_{i j}^{l} \leq 0 \leq b_{j}^{l}+M \quad$ and $\quad b_{j}^{l}-2 M<-\bar{v}_{i j}^{k}+\bar{v}_{i j}^{l} \leq 0 \quad ; \quad$ by $a d_{i j}^{l k}=0$, it can be guaranteed that $-\bar{v}_{i j}^{k}+\bar{v}_{i j}^{l} \leq 0 \leq b_{j}^{l}+2 M$ and $b_{j}^{l}-M<-\bar{v}_{i j}^{k}+\bar{v}_{i j}^{l} \leq 0$; (6) When $y_{i j}^{l k}=0$, based on (2), $-\bar{v}_{i j}^{k}+\bar{v}_{i j}^{l}>0$. Then, $a d_{i j}^{l k}=1$ guarantees $0<-\bar{v}_{i j}^{k}+\bar{v}_{i j}^{l} \leq b_{j}^{l}$ and $b_{j}^{l}-M<0<-\bar{v}_{i j}^{k}+\bar{v}_{i j}^{l} ;$ while $a d_{i j}^{l k}=0$ guarantees $0<-\bar{v}_{i j}^{k}+\bar{v}_{i j}^{l} \leq b_{j}^{l}+M$ and $0<b_{j}^{l}<-\bar{v}_{i j}^{k}+\bar{v}_{i j}^{l}$.

Therefore, we have $a d_{i j}^{l k}=\left\{\begin{array}{ll}1 & \text { if } 0 \leq-\bar{v}_{i j}^{k}+\bar{v}_{i j}^{l} \leq b_{j}^{l} \\ 0 & \text { if }-\bar{v}_{i j}^{k}+\bar{v}_{i j}^{l}>b_{j}^{l}>0\end{array}\right.$.

Then, constraint (25) implies $a d_{i j}^{l k}=\left\{\begin{array}{ll}1 & \text { if }\left|\bar{v}_{i j}^{k}-\bar{v}_{i j}^{l}\right| \leq b_{j}^{l} \\ 0 & \text { if }\left|\bar{v}_{i j}^{k}-\bar{v}_{i j}^{l}\right|>b_{j}^{l}\end{array}\right.$.

This completes the Proof of Lemma 1.

Proposition 1: Let $M$ be a large enough number. The model (17) can be transformed into the below 0-1 mixed linear programming model (29).

$$
\begin{array}{lll}
\min -\frac{1}{m n r(r-1)} \sum_{l=1, l \neq k}^{r} \sum_{k=1}^{r} \sum_{i=1}^{m} \sum_{j=1}^{n} r d_{i j}^{l k} & \\
\text { s.t. } & \bar{v}_{i j}^{k}-v_{i j}^{k} \leq b_{j}^{k} & i=1,2, \cdots, m ; j=1,2, \cdots, n ; \\
& k=1,2, \cdots, r \\
\bar{v}_{i j}^{k}-v_{i j}^{k} \geq-b_{j}^{k} & i=1,2, \cdots, m ; j=1,2, \cdots, n ; \\
& k=1,2, \cdots, r \\
\bar{v}_{i j}^{k}-\bar{v}_{i j}^{l} \geq\left(y_{i j}^{l k}-1\right) M & i=1,2, \cdots, m ; j=1,2, \cdots, n ; \\
& l, k=1,2, \cdots, r ; l \neq k \\
\bar{v}_{i j}^{k}-\bar{v}_{i j}^{l}<y_{i j}^{l k} M & i=1,2, \cdots, m ; j=1,2, \cdots, n ; \\
& l, k=1,2, \cdots, r ; l \neq k \\
\bar{v}_{i j}^{k}-\bar{v}_{i j}^{l} \leq b_{j}^{l}+\left(2-y_{i j}^{l k}-a d_{i j}^{l k}\right) M & i=1,2, \cdots, m ; j=1,2, \cdots, n ; \\
& l, k=1,2, \cdots, r ; l \neq k \\
\bar{v}_{i j}^{k}-\bar{v}_{i j}^{l}>b_{j}^{l}-\left(1-y_{i j}^{l k}+a d_{i j}^{l k}\right) M & i=1,2, \cdots, m ; j=1,2, \cdots, n ; \\
-\bar{v}_{i j}^{k}+\bar{v}_{i j}^{l} \leq b_{j}^{l}+\left(y_{i j}^{l k}+1-a d_{i j}^{l k}\right) M & l, k=1,2, \cdots, r ; l \neq k \\
-\bar{v}_{i j}^{k}+\bar{v}_{i j}^{l}>b_{j}^{l}-\left(y_{i j}^{l k}+a d_{i j}^{l k}\right) M & i=1,2, \cdots, m ; j=1,2, \cdots, n ; \\
0 \leq \bar{v}_{i j}^{k} \leq 1 & i=1,2, \cdots, m ; j=1,2, \cdots, n ; \\
& l, k=1,2, \cdots, r ; l \neq k \\
y_{i j}^{l k}, a d_{i j}^{l k} \in\{0,1\} & i=1,2, \cdots, m ; j=1,2, \cdots, n ; \\
& k=1,2, \cdots, r
\end{array}
$$

Proposition 2: Let $f_{i j}^{k}=\bar{v}_{i j}^{k}-v_{i j}^{k}, u_{i j}^{k}=\left|f_{i j}^{k}\right|$, and $M$ be a large enough number. Then model (20) can be transformed into the below 0-1 mixed linear programming model (30).

$$
\begin{aligned}
& \min \frac{1}{r m n} \sum_{k=1}^{r} \sum_{i=1}^{m} \sum_{j=1}^{n} u_{i j}^{k}
\end{aligned}
$$

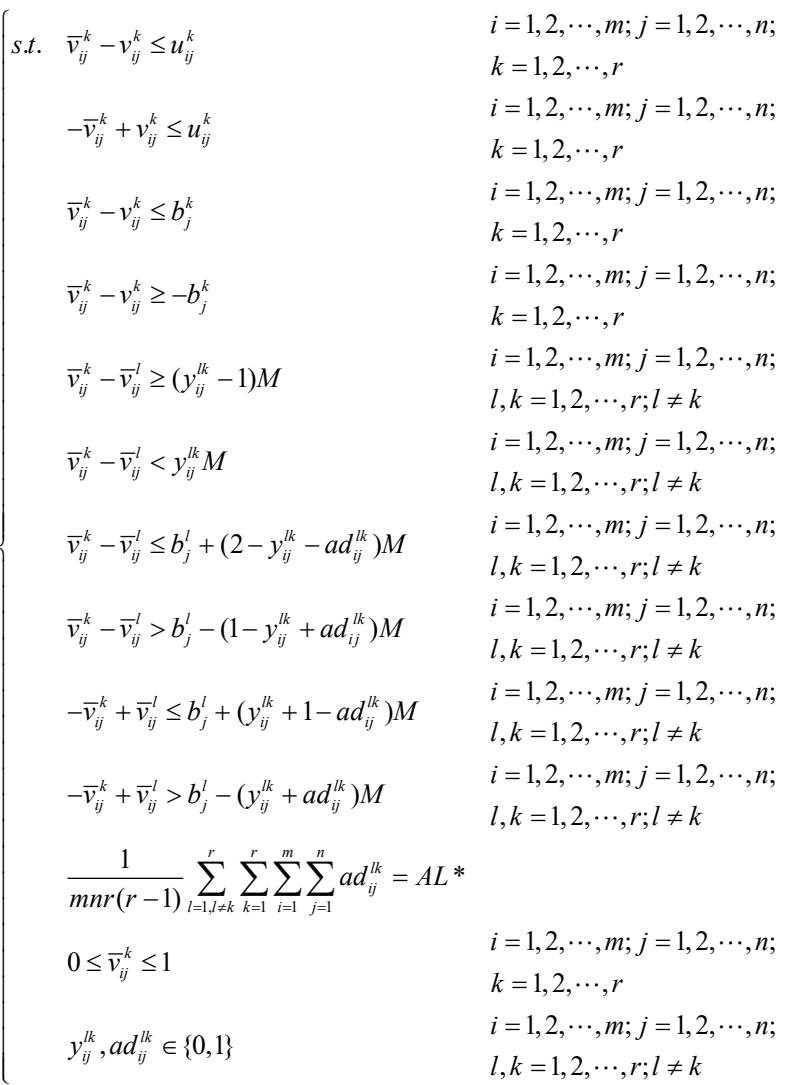

Proof: The main part of the proof of Propositions 1 and 2 can be obtained from the proof of Lemma 1, so the detailed description is omitted.

\section{E. Algorithm for the MOFMCM Model}

Based on the above descriptions, the framework of MOFMCM model is detailed in Algorithm I. Due to the time limitation in the actual consensus process, the maximum number of iterations of Algorithm I usually does not exceed 5 rounds [27], [30]. Therefore, the key to solving the computational complexity of Algorithm I depends on its linear programming models. Dantzig [55] pointed out that the average time complexity of the simplex algorithm to solve linear programming model is $O(n)$, which shows that the average time complexity of our linear programming models using Dantzig's method is also $O(n)$.

\section{Algorithm 1: MOFMCM model}

Input: Initial decision matrices $\left\{V^{1}, V^{2}, \cdots, V^{r}\right\}$, individual bounded confidences $\left\{B^{1}, B^{2}, \cdots, B^{r}\right\}$, mutual acceptance threshold $\mu$, decision makers' weights $\left\{\omega_{1}, \omega_{2}, \cdots, \omega_{m}\right\}$, attributes' weights $\left\{w_{1}, w_{2}, \cdots, w_{n}\right\}$.

Output: The ranking of alternatives.

Step 1: Let $t=0$ and $V^{k, t}=\left(v_{i j}^{k, t}\right)_{m \times n}=\left(v_{i j}^{k}\right)_{m \times n}(k=1,2, \cdots, r)$.

Step 2: Using Eqs.(10)-(12), obtain the group acceptance level of decision make $d_{k}(k=1,2, \cdots, r)$ at round $t \quad A L^{t}\left(d_{k}\right)=\sum_{l=1, l \neq k}^{r} A L^{l k \neq} /(r-1)$, where $A L^{l k, t}=\frac{\sum_{i=1}^{m} \sum_{j=1}^{n} a d_{i j}^{l k, t}}{m n}$ and $a d_{i j}^{l k, t}=\left\{\begin{array}{ll}1 & \text { if }\left|v_{i j}^{k, t}-v_{i j}^{l, t}\right| \leq b_{j}^{l} \\ 0 & \text { if }\left|v_{i j}^{k, t}-v_{i j}^{l, t}\right|>b_{j}^{l}\end{array}\right.$.

Step 3: Apply Eq. (10) to compute the group mutual acceptance at round $t$ : 


$$
A L^{t}=\sum_{k=1}^{r} A L^{t}\left(d_{k}\right) / r
$$

Step 4: If $A L^{t}<\mu$, i.e., $A L^{t}$ at round $t$ is unacceptable, then go to Step 5; otherwise, go to Step 9.

Step 5: Solve model (17) and obtain $A L^{t *}$. If $A L^{t *}=A L^{t}$, then go to Step 7; otherwise, continue Step 6

Step 6: Solve model (20) and obtain $\bar{V}^{k, t *}(k=1,2, \cdots, r)$. The recommended adjusted decision matrix $V^{k, t+1}=\left(v_{i j}^{k, t+1}\right)_{m \times n}$ follow the rule: $v_{i j}^{k, t+1} \in\left[\min \left(v_{i j}^{k, t}, \bar{v}_{i j}^{k, t *}\right), \max \left(v_{i j}^{k, t}, \bar{v}_{i j}^{k, t *}\right)\right]$. Go to Step 8.

Step 7: Solve model (23) to obtain $\bar{V}^{k, t * *}$ and $\bar{V}^{c, t * *}$. Compute $C L^{t *}$ based on Eq. (24). Solve model (26) to obtain $\bar{V}^{k, t * *^{\prime}}(k=1,2, \cdots, r)$. The recommended adjusted decision matrices $V^{k, t+1}=\left(v_{i j}^{k, t+1}\right)_{m \times n}$ follow the rule: $v_{i j}^{k, t+1} \in\left[\min \left(v_{i j}^{k, t}, v_{i j}^{k, t * *^{\prime}}\right), \max \left(v_{i j}^{k, t}, \vec{v}_{i j}^{k, t * *^{\prime}}\right)\right]$.

Step 8: Let $t=t+1$; and go back to Step 2 .

Step 9: Apply Eq. (1) to aggregate $\left\{V^{1, t}, V^{2, t}, \cdots, V^{r, t}\right\}$ into $V^{c, t}=\left(v_{i j}^{c, t}\right)_{m \times n}$, where $v_{i j}^{c, t}=\sum_{k=1}^{r} \omega_{k} v_{i j}^{k, t}$. Then, output the ranking of alternatives derived from the dominance value $Q_{i}=\sum_{j=1}^{n} w_{j} c_{i j}^{c, t}$.

\section{EXPERIMENTAL ANALYSIS}

A numerical analysis is included first in this section to illustrate the usage of the MOFMCM model. Then, simulation analysis I is proposed to study the influence of the bounded confidence on the MOFMCM model, while simulation analysis II is designed to compare the consensus effectiveness of the MOFMCM model and the general consensus reaching model (abbreviated as the GCR).

\section{A. Numerical Analysis}

A gearbox manufacturing enterprise needs EPM software to be supplied by one of four software suppliers $X=\left\{x_{1}, x_{2}, x_{3}, x_{4}\right\}$. A manager and five experts from different departments (information; project management; financial; planning; collaborators) use four qualitative attributes to evaluate and compare the four suppliers: after-sales service and training $\left(a_{1}\right)$, core function $\left(a_{2}\right)$, technical support level $\left(a_{3}\right)$ and software cost $\left(a_{4}\right)$. The weights associated with the experts and attributes are $\omega=\{1 / 4,1 / 4, \cdots, 1 / 4\} \quad$ and $w=\{0.15,0.25,0.2,0.4\}$, respectively. In this MAGDM problem, the mutual acceptance threshold is set as $\mu=0.8$, while the individual bounded confidences and initial decision matrices are listed in Tables 1 and 2, respectively. Using Eq. (1), the collective decision matrix of initial decision matrices, $V^{c, 0}$, is obtained as shown in Table 3. Applying Eq. (5), we can obtain the dominance values of alternatives: $Q_{1}=0.513$, $Q_{2}=0.464, Q_{3}=0.512, Q_{4}=0.475$. Then, the corresponding initial alternative ordering is: $x_{1} \succ x_{3} \succ x_{4} \succ x_{2}$.

Table 1. individual bounded confidences $B^{k}(k=1,2, \cdots, 4)$
\begin{tabular}{cccccc}
\hline & $a_{1}$ & $a_{2}$ & $a_{3}$ & $a_{4}$ \\
\hline$B^{1}$ & 0.36 & 0.35 & 0.30 & 0.32 \\
$B^{2}$ & 0.35 & 0.40 & 0.43 & 0.45 \\
$B^{3}$ & 0.30 & 0.34 & 0.28 & 0.27 \\
$B^{4}$ & 0.05 & 0.20 & 0.15 & 0.13 \\
\hline
\end{tabular}

Table 2. initial decision matrices $V^{k}(k=1,2, \cdots, 4)$

\begin{tabular}{|c|c|c|c|c|c|c|c|c|}
\hline & $a_{1}$ & $a_{2}$ & $a_{3}$ & $a_{4}$ & $a_{1}$ & $a_{2}$ & $a_{3}$ & $a_{4}$ \\
\hline & \multicolumn{4}{|c|}{$d_{1}$} & \multicolumn{4}{|c|}{$d_{2}$} \\
\hline$x_{1}$ & 0.45 & 0.90 & 0.70 & 0.75 & 0.65 & 0.20 & 0.20 & 0.60 \\
\hline$x_{2}$ & 0.65 & 0.50 & 0.45 & 0.80 & 0.10 & 0.30 & 0.50 & 0.55 \\
\hline$x_{3}$ & 0.80 & 0.25 & 0.20 & 0.80 & 0.20 & 0.60 & 0.25 & 0.20 \\
\hline \multirow[t]{2}{*}{$x_{4}$} & 0.90 & 0.15 & 0.90 & 0.65 & 0.30 & 0.50 & 0.85 & 0.45 \\
\hline & \multicolumn{4}{|c|}{$d_{3}$} & \multicolumn{4}{|c|}{$d_{4}$} \\
\hline$x_{1}$ & 0.50 & 0.55 & 0.40 & 0.45 & 0.30 & 0.50 & 0.65 & 0.30 \\
\hline$x_{2}$ & 0.30 & 0.15 & 0.30 & 0.55 & 0.50 & 0.70 & 0.10 & 0.45 \\
\hline$x_{3}$ & 0.70 & 0.65 & 0.55 & 0.75 & 0.35 & 0.70 & 0.75 & 0.35 \\
\hline$x_{4}$ & 0.65 & 0.40 & 0.55 & 0.45 & 0.95 & 0.15 & 0.30 & 0.10 \\
\hline
\end{tabular}

Table 3. The collective decision matrix $V^{c, 0}$

\begin{tabular}{ccccc}
\hline \hline & $a_{1}$ & $a_{2}$ & $a_{3}$ & $a_{4}$ \\
\hline$x_{1}$ & 0.48 & 0.54 & 0.49 & 0.53 \\
$x_{2}$ & 0.39 & 0.41 & 0.34 & 0.59 \\
$x_{3}$ & 0.51 & 0.55 & 0.44 & 0.53 \\
$x_{4}$ & 0.70 & 0.30 & 0.65 & 0.41 \\
\hline
\end{tabular}

(1) First round: Using Eqs. (10)-(13), the group mutual acceptance is obtained: $A L^{1}=0.57$. The experts are advised to adjust decision matrices since $A L^{1}<\mu$. Solving model (17),

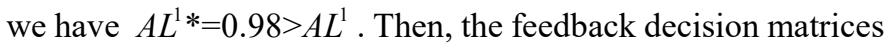
$\bar{V}^{k, 1} *$ listed in Table 4 are obtained by solving model (20).

Table 4 . The feedback decision matrices $\bar{V}^{k, 1} *$ at first round

\begin{tabular}{|c|c|c|c|c|c|c|c|c|}
\hline & $a_{1}$ & $a_{2}$ & $a_{3}$ & $a_{4}$ & $a_{1}$ & $a_{2}$ & $a_{3}$ & $a_{4}$ \\
\hline & \multicolumn{4}{|c|}{$d_{1}$} & \multicolumn{4}{|c|}{$d_{2}$} \\
\hline$x_{1}$ & 0.45 & 0.65 & 0.65 & 0.60 & 0.55 & 0.35 & 0.45 & 0.60 \\
\hline$x_{2}$ & 0.50 & 0.50 & 0.30 & 0.75 & 0.25 & 0.40 & 0.30 & 0.55 \\
\hline$x_{3}$ & 0.60 & 0.50 & 0.35 & 0.65 & 0.35 & 0.60 & 0.55 & 0.33 \\
\hline \multirow[t]{2}{*}{$x_{4}$} & 0.90 & 0.15 & 0.70 & 0.33 & 0.65 & 0.15 & 0.73 & 0.45 \\
\hline & \multicolumn{4}{|c|}{$d_{3}$} & \multicolumn{4}{|c|}{$d_{4}$} \\
\hline$x_{1}$ & 0.50 & 0.55 & 0.45 & 0.45 & 0.30 & 0.50 & 0.55 & 0.30 \\
\hline$x_{2}$ & 0.30 & 0.40 & 0.30 & 0.55 & 0.50 & 0.55 & 0.20 & 0.45 \\
\hline$x_{3}$ & 0.60 & 0.65 & 0.55 & 0.65 & 0.35 & 0.65 & 0.65 & 0.35 \\
\hline$x_{4}$ & 0.65 & 0.30 & 0.50 & 0.45 & 0.90 & 0.15 & 0.40 & 0.33 \\
\hline
\end{tabular}

(2) Second round: Based on the feedback recommendation, the decision matrices are adjusted to the ones of Table 5. The mutual acceptance at this round is still below the threshold: $A L^{2}=0.75<\mu$. Maximum mutual acceptance $A L^{2} *=1>A L^{2}$ is obtained by solving model (17). Solving model (23), we have the optimal solution $\bar{V}^{k, 2 *}$ of Table 6 .

(3) Third round: At this round, the adjusted decision matrices $V^{k, 3}$ are obtained and listed in Table 7 leading to a satisfactory mutual acceptance: $A L^{3}=0.802>\mu=0.8$. Applying Eq. (1), the collective decision matrix $V^{c, 3}$ is obtained (Table 8), from which the dominance values of alternatives are obtained applying Eq. (5): $Q_{1}=0.497, Q_{2}=0.460, Q_{3}=0.543$, 
$Q_{4}=0.412$. Then, we have the alternative ordering $x_{3} \succ x_{1} \succ x_{2} \succ x_{4}$, and the gearbox manufacturing enterprise ultimately choose $x_{3}$ as its software supplier.

Table 5. The adjusted decision matrices $V^{k, 2}$

\begin{tabular}{|c|c|c|c|c|c|c|c|c|}
\hline & $a_{1}$ & $a_{2}$ & $a_{3}$ & $a_{4}$ & $a_{1}$ & $a_{2}$ & $a_{3}$ & $a_{4}$ \\
\hline & \multicolumn{4}{|c|}{$d_{1}$} & \multicolumn{4}{|c|}{$d_{2}$} \\
\hline$x_{1}$ & 0.45 & 0.70 & 0.66 & 0.63 & 0.57 & 0.32 & 0.40 & 0.60 \\
\hline$x_{2}$ & 0.53 & 0.50 & 0.33 & 0.76 & 0.22 & 0.38 & 0.34 & 0.55 \\
\hline$x_{3}$ & 0.64 & 0.45 & 0.32 & 0.68 & 0.32 & 0.60 & 0.49 & 0.30 \\
\hline \multirow[t]{2}{*}{$x_{4}$} & 0.90 & 0.15 & 0.74 & 0.39 & 0.58 & 0.22 & 0.75 & 0.45 \\
\hline & \multicolumn{4}{|c|}{$d_{3}$} & \multicolumn{4}{|c|}{$d_{4}$} \\
\hline$x_{1}$ & 0.50 & 0.55 & 0.43 & 0.45 & 0.30 & 0.50 & 0.63 & 0.30 \\
\hline$x_{2}$ & 0.30 & 0.31 & 0.30 & 0.55 & 0.50 & 0.67 & 0.12 & 0.45 \\
\hline$x_{3}$ & 0.64 & 0.65 & 0.55 & 0.69 & 0.35 & 0.69 & 0.73 & 0.35 \\
\hline$x_{4}$ & 0.65 & 0.34 & 0.52 & 0.45 & 0.94 & 0.15 & 0.32 & 0.15 \\
\hline
\end{tabular}

Table 6 . The feedback decision matrices $\bar{V}^{k, 2 *}$ at second round

\begin{tabular}{|c|c|c|c|c|c|c|c|c|}
\hline & $a_{1}$ & $a_{2}$ & $a_{3}$ & $a_{4}$ & $a_{1}$ & $a_{2}$ & $a_{3}$ & $a_{4}$ \\
\hline & \multicolumn{4}{|c|}{$d_{1}$} & \multicolumn{4}{|c|}{$d_{2}$} \\
\hline$x_{1}$ & 0.45 & 0.65 & 0.63 & 0.60 & 0.55 & 0.35 & 0.43 & 0.60 \\
\hline$x_{2}$ & 0.50 & 0.50 & 0.32 & 0.75 & 0.25 & 0.38 & 0.32 & 0.55 \\
\hline$x_{3}$ & 0.63 & 0.45 & 0.53 & 0.68 & 0.38 & 0.60 & 0.53 & 0.36 \\
\hline \multirow[t]{2}{*}{$x_{4}$} & 0.90 & 0.15 & 0.52 & 0.39 & 0.65 & 0.22 & 0.52 & 0.45 \\
\hline & \multicolumn{4}{|c|}{$d_{3}$} & \multicolumn{4}{|c|}{$d_{4}$} \\
\hline$x_{1}$ & 0.50 & 0.55 & 0.43 & 0.45 & 0.30 & 0.50 & 0.53 & 0.30 \\
\hline$x_{2}$ & 0.30 & 0.38 & 0.30 & 0.55 & 0.50 & 0.53 & 0.22 & 0.45 \\
\hline$x_{3}$ & 0.64 & 0.65 & 0.55 & 0.68 & 0.38 & 0.60 & 0.63 & 0.38 \\
\hline$x_{4}$ & 0.65 & 0.30 & 0.52 & 0.45 & 0.90 & 0.15 & 0.42 & 0.15 \\
\hline \multicolumn{9}{|c|}{ Table 7 . The adjusted decision matrices $V^{k, 3}$} \\
\hline & $a_{1}$ & $a_{2}$ & $a_{3}$ & $a_{4}$ & $a_{1}$ & $a_{2}$ & $a_{3}$ & $a_{4}$ \\
\hline & \multicolumn{4}{|c|}{$d_{1}$} & \multicolumn{4}{|c|}{$d_{2}$} \\
\hline$x_{1}$ & 0.45 & 0.66 & 0.64 & 0.61 & 0.55 & 0.34 & 0.43 & 0.60 \\
\hline$x_{2}$ & 0.51 & 0.50 & 0.32 & 0.75 & 0.24 & 0.38 & 0.32 & 0.55 \\
\hline$x_{3}$ & 0.64 & 0.45 & 0.49 & 0.68 & 0.37 & 0.60 & 0.52 & 0.35 \\
\hline$x_{4}$ & 0.90 & 0.15 & 0.56 & 0.39 & 0.64 & 0.22 & 0.57 & 0.45 \\
\hline & \multicolumn{4}{|c|}{$d_{3}$} & \multicolumn{4}{|c|}{$d_{4}$} \\
\hline$x_{1}$ & 0.50 & 0.55 & 0.43 & 0.45 & 0.30 & 0.50 & 0.61 & 0.30 \\
\hline$x_{2}$ & 0.30 & 0.36 & 0.30 & 0.55 & 0.50 & 0.64 & 0.14 & 0.45 \\
\hline$x_{3}$ & 0.64 & 0.65 & 0.55 & 0.68 & 0.36 & 0.67 & 0.71 & 0.36 \\
\hline$x_{4}$ & 0.65 & 0.31 & 0.52 & 0.45 & 0.93 & 0.15 & 0.34 & 0.15 \\
\hline
\end{tabular}

Table 8 . The collective decision matrix $V^{c, 3}$

\begin{tabular}{ccccc}
\hline & $a_{1}$ & $a_{2}$ & $a_{3}$ & $a_{4}$ \\
\hline$x_{1}$ & 0.45 & 0.51 & 0.53 & 0.49 \\
$x_{2}$ & 0.39 & 0.47 & 0.27 & 0.58 \\
$x_{3}$ & 0.50 & 0.59 & 0.57 & 0.52 \\
$x_{4}$ & 0.78 & 0.21 & 0.50 & 0.36 \\
\hline \hline
\end{tabular}

\section{B. Simulation Analysis I}

Simulation analysis I is designed to study the influence of the bounded confidence on the consensus effectiveness of the MOFMCM model. In this simulation, the decision matrices are randomly generated with initial values. Specifically, the settings and issues involved in Simulation analysis I are as follows:

(1) The bounded confidences are set within $\left[\delta_{\min }, \delta_{\max }\right]$, namely $b_{j}^{k} \in\left[\delta_{\min }, \delta_{\max }\right]$, with the aim of studying the influence of different levels of bounded confidence on consensus reaching.

(2) To automatically obtain the adjusted decision matrices without changing the essence of the MOFMCM model, Eqs. (21) and (27) are replaced with Eqs. (31) and (32), respectively.

$$
\begin{gathered}
v_{i j}^{k^{\prime}}=\alpha_{k} v_{i j}^{k}+\left(1-\alpha_{k}\right) \bar{v}_{i j}^{k *}(i=1, \cdots, m ; j=1, \cdots, n) \\
v_{i j}^{k^{\prime}}=\alpha_{k} v_{i j}^{k}+\left(1-\alpha_{k}\right) \bar{v}_{i j}^{k} * *^{\prime}(i=1, \cdots, m ; j=1, \cdots, n)
\end{gathered}
$$

where $\alpha_{k} \in[0,1]$ is a randomly generated parameter to derive feedback decision matrices.

Let $r=5$, and $T=5$. We run Simulation analysis I 1000 times to obtain the mutual acceptance average value, $A L^{t}$, under different levels of $\left[\delta_{\min }, \delta_{\max }\right]$. The obtained results are shown in Fig. 3, from where it is observed that the values of $A L^{t}$ increase when the values of interval $\left[\delta_{\min }, \delta_{\max }\right]$ increase (The same is true at $t=0)$. This indicates that the group will achieve a higher mutual acceptance level when the decision makers are more acceptable on the attributes.

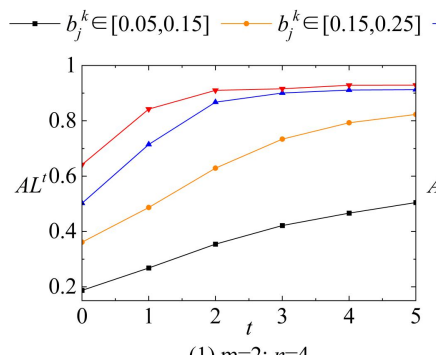

$$
\longrightarrow b_{j}^{k} \in[0.25,0.35] \longrightarrow b_{j}^{k} \in[0.35,0.45]
$$
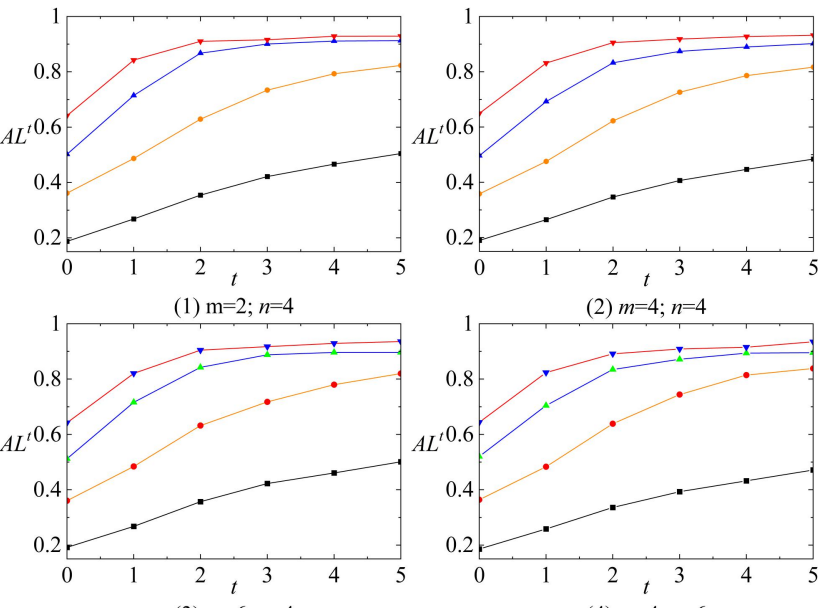

(3) $m=6 ; n=4$

(4) $m=4 ; n=6$

Fig. 3. The mutual acceptance $A L^{t}$ under different levels of interval

$$
\left[\delta_{\min }, \delta_{\max }\right]
$$

Simulation analysis $\mathbf{I}$ : The influence of bounded confidence on the MOFMCM model

Input: the number of decision makers $r$, the number of alternative $m$, the number of attributes $n$, the maximum number of rounds $T$, the bounded confidence interval $\left[\delta_{\min }, \delta_{\max }\right]$.

Output: the mutual acceptance in each round $A L^{t}(t=0,1,2 \cdots)$.

Steps 2, 3, 5 and 8 are the same as those of Algorithm 1, and the other steps are described below. 
Step 1: Uniformly and randomly generate $v_{i j}^{k}$ of $V^{k}=\left(v_{i j}^{k}\right)_{m \times n}(k=1,2, \cdots, r)$ from $[0,1]$ and $b_{j}^{k}(j=1,2, \cdots, n)$ from $\left[\delta_{\min }, \delta_{\max }\right]$. Let $t=0$ and $V^{k, t}=\left(v_{i j}^{k, t}\right)_{m \times n}=\left(v_{i j}^{k}\right)_{m \times n}$.

Step 4: If $t<T$, run Step 5; otherwise, run Step 9.

Step 6: Solve model (17) to derive optimal solution $\bar{V}^{k, t *}$. Based on Eq. (31), generate the adjusted decision matrices $V^{k, t+1}=\left(v_{i j}^{k, t+1}\right)_{m \times n}$, where $v_{i j}^{k, t+1}=\alpha_{k} v_{i j}^{k, t}+\left(1-\alpha_{k}\right) \bar{v}_{i j}^{k, t} *$. Go to Step 8.

Step 7: Solve model (23) to obtain $\bar{V}^{k, t * *}$ and $\bar{V}^{c, t * *}$. Obtain $C L^{t *}$ using Eq. (24). Solve model (26) to obtain $\bar{V}^{k, t * *^{\prime}}$. Use Eq. (31) to generate the adjusted decision matrix $V^{k, t+1}=\left(v_{i j}^{k, t+1}\right)_{m \times n}$, where

$v_{i j}^{k, t+1}=\alpha_{k} v_{i j}^{k, t}+\left(1-\alpha_{k}\right) \bar{v}_{i j}^{k, t} * *^{\prime}$.

Step 9: Output the mutual acceptance $A L^{t}(t=0,1,2 \cdots)$.

\section{Simulation Analysis II}

Simulation analysis II is used to compare the consensus effectiveness of the MOFMCM model and the GCR model, in which the initial decision matrices are still randomly generated. And the effectiveness is represented by the following two indicators:

(1) The mutual acceptance level $A L^{t}$ at each round;

(2) The preference adjustment $P A^{t}$ at each round, as per the following expression: $P A^{t}=\sum_{k=1}^{r} \sum_{i=1}^{m} \sum_{j=1}^{n}\left|v_{i j}^{k, t}-v_{i j}^{k, t-1}\right|$.

Simulation analysis II is a modified version of Simulation analysis I to obtain the preference adjustment $P A^{t}$, with modifications being:

(1) Add computation of the preference adjustment $P A^{t}$ at Steps 6 and 7.

(2) Step 9 is modified to: Output the mutual acceptance $A L^{t}$ and the preference adjustment $P A^{t}$.

Simulation experiment II' is used to analyze the effectiveness of the GCR model, which follows the framework of the general consensus process shown of Fig. 1. To align the GCR model with the MOFMCM model, IR of the GCR model identifies all decision makers. Simulation experiment II' is also a modified version of Simulation analysis I, by replacing Steps 4-9 with Steps 4'-6' below.

Steps 4': If $t<T$, continue Step 5'; otherwise, go to Step 6'.

Steps 5': Using Eq. (1) compute $V^{c, t}$, where $v_{i j}^{c, t}=\sum_{k=1}^{r} \omega_{k} v_{i j}^{k, t}$. Obtain the adjusted decision matrices $V^{k, t+1}=\left(v_{i j}^{k, t+1}\right)_{m \times n}$, where

$$
\left\{\begin{array}{ll}
v_{i j}^{k, t+1}=\alpha_{k} v_{i j}^{k, t}+\left(1-\alpha_{k}\right) v_{i j}^{c, t} & \text { if } b_{j}^{k} \geq\left|v_{i j}^{k, t}-v_{i j}^{\mathrm{c}, t}\right| \\
v_{i j}^{k, t+1}=v_{i j}^{k, t} & \text { if } b_{j}^{k} \triangleleft v_{i j}^{k, t}-v_{i j}^{\mathrm{c}, t} \mid
\end{array} .\right.
$$

Compute $P A^{t+1}=\sum_{k=1}^{r} \sum_{i=1}^{m} \sum_{j=1}^{n}\left|v_{i j}^{k, t+1}-v_{i j}^{k, t}\right|$. Let $t=t+1 ;$ and go to Step 2.

Steps 6': Output the mutual acceptance $A L^{t}$ and the preference adjustment $P A^{t}$.

Let $r=5, m=5, n=4$, and $T=5$. We run simulation analyses II and II' 1000 times to obtain the average values of the mutual acceptance $A L^{t}$ and the preference adjustment $P A^{t}$ under different bounded confidence intervals $\left[\delta_{\min }, \delta_{\max }\right]$. The simulation results are shown in Figs. 4 and 5, from which the following observations are drawn:
(1) From Figs. 4(1), 4(2), 4(4), 5(1), 5(2), and 5(4), the average values for $A L^{t}$ and $P A^{t}$ of the GCR model are lower than those of the MOFMCM model. This is because that the feedback recommendations generated by the GCR model are unacceptable for decision makers with low bounded confidence levels. And this resulted in them not changing their preferences, and the level of group mutual acceptance hardly increased. On the contrary, the feedback recommendations of the MOFMCM model are accepted by the decision makers, thereby adjusting their preferences and increasing the level of group mutual acceptance.

(2) For a large bounded confidence interval $\left[\delta_{\text {min }}, \delta_{\max }\right]$ (see Fig. 4(3) and Fig. 5(3)), the average values for $A L^{t}$ of the GCR model are lower than those of the MOFMCM model, while the average values for $P A^{t}$ of the GCR model are greater than those of the MOFMCM model. Therefore, compared with the GCR, the MOFMCM can reach a higher level of mutual acceptance more efficiently.
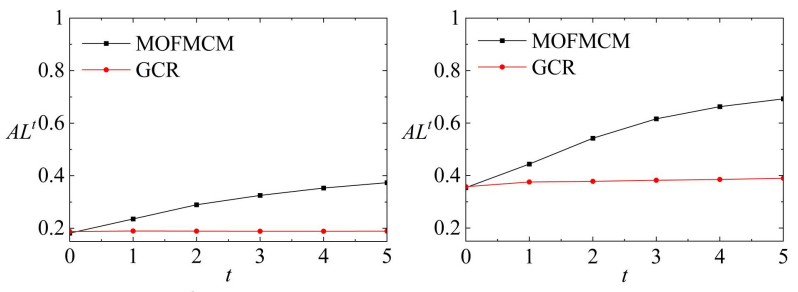

(1) $b_{j}^{k} \in[0.05,0.15]$
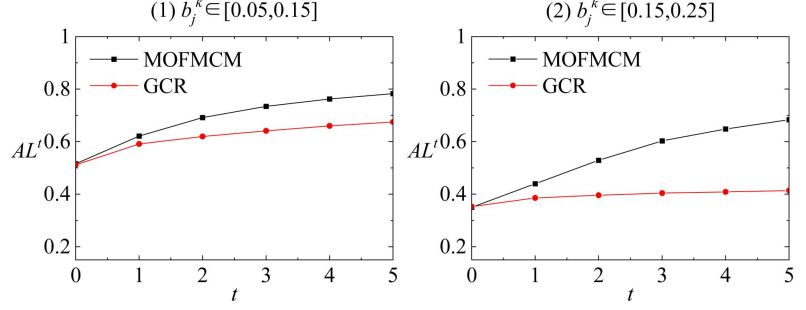

(3) $b_{j}^{k} \in[0.25,0.35]$

(4) $b_{j}^{k} \in[0.05,0.35]$

Fig. 4. Average $A L^{t}$ values in MOFMCM and GCR models under different bounded confidence intervals $\left[\delta_{\min }, \delta_{\max }\right]$
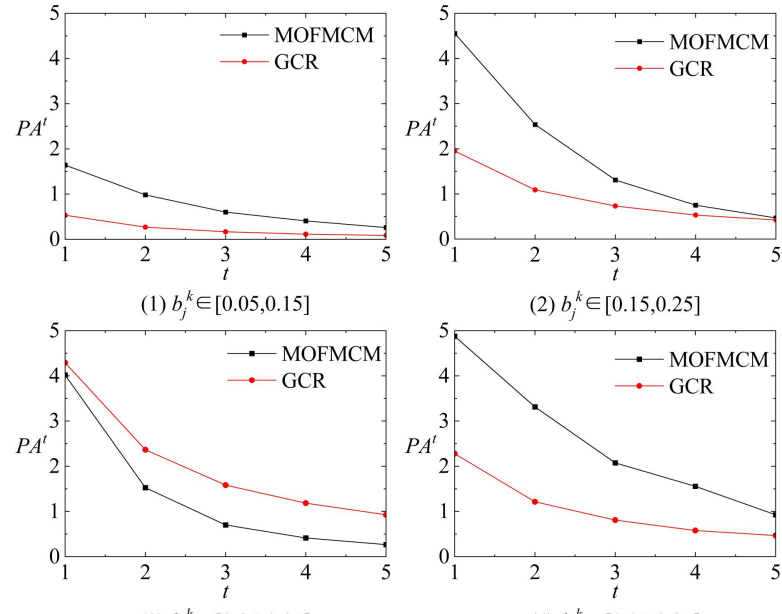

(2) $b_{j}^{k} \in[0.15,0.25]$

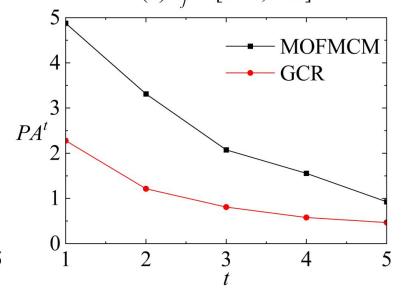

(4) $b_{j}^{k} \in[0.05,0.35]$

Fig. 5. Average $P A^{t}$ values in MOFMCM and GCR models under different bounded confidence intervals $\left[\delta_{\min }, \delta_{\max }\right]$

$T$ is the number of iterations in simulation analysis, usually no more than 5 rounds in practical consensus processes. Based on the description of computational complexity before, we have that the average time complexity of our linear programming 
models in Simulation analyses I and II is $O(n)$ by the Dantzig's method [55]. Furthermore, computational complexity is generally considered in the worst case, in which, the GCR model can not converge to consensus, while the MOFMCM model can achieve. The reason is that the GCP model may always produces unacceptable feedback recommendations as shown in Fig .4(1), and the MOFMCM model produces acceptable feedback recommendations based on bounded confidence. In summary, the feedback recommendations of the MOFMCM model are easier to be accepted to promote the group mutual acceptance improvement.

\section{CONCLUSION}

This paper proposed a multi-stage optimization feedback mechanism based consensus model in MAGDM that aims to help decision makers improve the level of group mutual acceptance based on their individual bounded confidences. The MOFMCM model is based on the group consensus level being related to the level of mutual acceptance, and measures the consensus level from the perspective of the mutual acceptance based on individual bounded confidences. The multi-stage optimization feedback mechanism of MOFMCM considers the willingness of decision makers to accept recommendations from the perspective of bounded confidence. In comparison with the similarity based feedback mechanisms, the priority here is given to a two-stage optimization from the perspective of mutual acceptance. Furthermore, the effectiveness of the MOFMCM model is verified through simulation and comparison analyses.

The social network has been studied widely in GDM in recent years [1], [16], [17], [23], [25], [26], [39]. However, these studies rarely combine social networks with individual bounded confidences for research. Our future work will focus on investigating the evolution of the preferences in GDM, based not only on the bounded confidences of the decision makers, but also on the existence of social relationships between decision makers.

\section{REFERENCES}

[1] B.W. Zhang, Y.C. Dong, H.J. Zhang, and W. Pedrycz, "Consensus Mechanism with Maximum-Return Modifications and Minimum-Cost Feedback: A Perspective of Game Theory," Eur. J. Oper. Res., vol. 287, pp. 546-559, 2020.

[2] M. Fedrizzi, J. Kacprzyk, and S. Zadrożny, "An interactive multi-user decision support system for consensus reaching processes using fuzzy logic with linguistic quantifiers," Decis. Support Syst., vol. 4, pp. 313-327, 1988.

[3] Z.W. Gong, X.X. Xu, H.H. Zhang, U. Aytun Ozturk, E. Herrera-Viedma, and $\mathrm{C} . \mathrm{Xu}$, "The consensus models with interval preference opinions and their economic interpretation," Omega, vol. 55, pp. 81-90, 2015.

[4] Z.W. Gong, H.H. Zhang, J. Forrest, L.S. Li, and X.X. Xu, "Two consensus models based on the minimum cost and maximum return regarding either all individuals or one individual,” Eur. J. Oper. Res., vol. 240, pp. 183-192, 2015.

[5] F.J. Cabrerizo, R. Al-Hmouz, A. Morfeq, A.S. Balamash, M.A. Martínez, and E. Herrera-Viedma, "Soft consensus measures in group decision making using unbalanced fuzzy linguistic information," Soft Comput., vol. 21, pp. 3037-3050, 2017.

[6] B. Spillman, J. Bezdek, and R. Spillman, "Development of an instrument for the dynamic measurement of consensus," Communication Monographs, vol. 46, pp. 1-12, 1979.
[7] F. Herrera, E. Herrera-Viedma, and J. Verdegay, "A model of consensus in group decision making under linguistic assessments," Fuzzy Sets Syst., vol. 78, pp. 73-87, 1996.

[8] D. Ben-Arieh and Z. Chen, "Linguistic group decision-making: opinion aggregation and measures of consensus," Fuzzy Optim. and Decis. Ma., vol. 5, pp. 371-386, 2006.

[9] J. Kacprzyk, M. Fedrizzi, "A 'soft' measure of consensus in the setting of partial (fuzzy) preferences," Eur. J. Oper. Res., vol. 34, pp. 316-325, 1988.

[10] F. Herrera, E. Herrera-Viedma, and J. Verdegay, "Linguistic measures based on fuzzy coincidence for reaching consensus in group decision making,” Int. J. Approx. Reason., vol. 16, pp. 309-334, 1997.

[11] J. Chen, C. Chen, C. Wang, X. Jiang, "Measuring soft consensus in uncertain linguistic group decision-making based on deviation and overlap degrees," International Journal of Innovative Management, Information \& Production, vol. 2, pp. 25-33, 2011.

[12] F.J. Cabrerizo, J.M. Moreno, I.J. Pérez, E. Herrera-Viedma, “Analyzing consensus approaches in fuzzy group decision making: advantages and drawbacks," Soft Comput., vol. 14, pp. 451-463, 2010.

[13] E. Herrera-Viedma, L. Martínez, F. Mata, F. Chiclana, "A consensus support system model for group decision-making problems with multigranular linguistic preference relations," IEEE Trans. Fuzzy Syst., vol. 13, pp. 644-658, 2005.

[14] E. Herrera-Viedma, F.J. Cabrerizo, J. Kacprzyk, and W. Pedrycz, "A review of soft consensus models in a fuzzy environment," Inf. Fusion, vol. 17, pp. 4-13, 2014.

[15] Z. Zhang and C.H. Guo, "Consistency and consensus models for group decision-making with uncertain 2-tuple linguistic preference relations," Int. J. of syst. Sci., vol. 47, pp. 2572-2587, 2016.

[16] Y.C. Dong, Q.B. Zha, H.J. Zhang, and F. Herrera, "Consensus reaching and strategic manipulation in group decision making with trust relationships," IEEE Trans. Syst., Man, Cybern., Syst., DOI: 10.1109/TSMC.2019.2961752

[17] J. Wu, F. Chiclana, H. Fujita, and E. Herrera-Viedma, "A visual interaction consensus model for social network group decision making with trust propagation,” Knowl. Based Syst., vol. 122, pp. 39-50, 2017.

[18] Y.C. Dong, Y.F. Xu, H.Y. Li, and B. Feng, "The OWA-based consensus operator under linguistic representation models using position indexes," Eur. J. Oper. Res., vol. 203, pp. 455-463, 2010.

[19] H.J. Zhang, Y.C. Dong, F. Chiclana, and S. Yu, "Consensus efficiency in group decision making: A comprehensive comparative study and its optimal design," Eur. J. Oper. Res., vol. 275, pp.580-598, 2019.

[20] W.J. Xu, X. Chen, Y.C. Dong, F. Chiclana, "Impact of decision rules and non-cooperative behaviors on minimum consensus cost in group decision making," Group Decis. Negotiation, Doi: 10.1007/s10726-020-09653-7.

[21] H.J. Zhang, Y.C. Dong, and X. Chen, "The 2-rank consensus reaching model in the multigranular linguistic multiple-attribute group decision-making," IEEE Trans. Syst., Man, Cybern., Syst., vol. 48, no. 12, pp. 2080-2094, 2018.

[22] D. Ben-Arieh and T. Easton, "Multi-criteria group consensus under linear cost opinion elasticity," Decis. Support Syst., vol. 43, pp. 713-721, 2007.

[23] J. Wu, L.F. Dai, F. Chiclana, H. Fujita, and E. Herrera-Viedma, "A minimum adjustment cost feedback mechanism based consensus model for group decision making under social network with distributed linguistic trust,” Inf. Fusion, vol. 41, pp. 232-242, 2017.

[24] Y. C. Dong, M. Zhan, G. Kou, Z. G. Ding, and H. M. Liang, "A survey on the fusion process in opinion dynamics," Inf. Fusion, vol. 43, pp. 57-5, Sep. 2018.

[25] R. Ureña, G. Kou, Y. C. Dong, F. Chiclana, and E. Herrera-Viedma, “A review on trust propagation and opinion dynamics in social networks and group decision making frameworks," Inf. Sci., vol. 478, pp. 461-475, Apr. 2019.

[26] Y.C. Dong, Q.B. Zha, H.J. Zhang, G. Kou, H. Fujita, F. Chiclana, and E. Herrera-Viedma, "Consensus reaching in social network group decision making: Research paradigms and challenges," Knowl. Based Syst., vol. 162, pp. 3-13, Dec. 2018.

[27] H.J. Zhang, J. Xiao, and Y.C. Dong, "Integrating a consensus-reaching mechanism with bounded confidences into failure mode and effect analysis under incomplete context," Knowl. Based Syst., vol. 183, 104873, 2019.

[28] H.M. Liang, Y.C. Dong, Z.G. Ding, R. Ureña, F. Chiclana, and E. Herrera-Viedma, "Consensus reaching with time constraints and minimum adjustments in group with bounded confifidence effects," IEEE Trans. Fuzzy Syst., Doi:10.1109/TFUZZ.2019.2939970.

[29] Q.B. Zha, Y.C. Dong, H.J. Zhang, F. Chiclana, and E. Herrera-Viedma, “A personalized feedback mechanism based on bounded confidence learning 
to support consensus reaching in group decision making," IEEE Trans. Syst., Man, Cybern., Syst., vol. 51, no. 6, 3900-3910, 2021.

[30] Q.B. Zha, H.M. Liang, G. Kou, Y.C. Dong, and S. Yu, “A feedback mechanism with bounded confidence-based optimization approach for consensus reaching in multiple attribute large-scale group decision making," IEEE Trans. on Comput. Soc. Syst., vol. 6, no. 5, 994-1006, 2019.

[31] F. Chiclana, J.M.T. Garcia, M.J. Del Moral, and E. Herrera-Viedma, “A statistical comparative study of different similarity measures of consensus in group decision making," Inf. Sci., vol. 221, pp. 110-123, Feb. 2013.

[32] R.R. Yager, "On ordered weighted averaging aggregation operators in multicriteria decision making," IEEE Trans. Syst., Man, Cybern., vol. 18, pp. 183-190, 1988.

[33] F. Herrera and E. Herrera-Viedma, "Choice functions and mechanisms for linguistic preference relations," Eur. J. Oper. Res., vol. 120, no. 1, pp. $144-161,2000$

[34] F. Chiclana, F. Herrera, and E. Herrera-Viedma, "Integrating multiplicative preference relations in a multipurpose decision-making model based on fuzzy preference relations," Fuzzy Sets Syst., vol. 122, pp. 277-291, 2001

[35] R. Hegselmann and U. Krause, "Opinion dynamics and bounded confidence models, analysis, and simulation,” J. Artif. Soc. Soc. Simulat., vol. 5, no. 3, pp. 1-33, 2002.

[36] G. Deffuant, D. Neau, F. Amblard, and G. Weisbuch, "Mixing beliefs among interacting agents," Adv. Complex Syst., vol. 3, no. 01n04, pp. $87-98,2000$.

[37] G. Weisbuch, G. Deffuant, F. Amblard, and J. P. Nadal, "Meet, discuss and segregate!" Complexity, vol. 7, no. 3, pp. 55-63, Feb. 2002.

[38] Y.J. Xu and N.N. Wu, "A two-stage consensus reaching model for group decision making with reciprocal fuzzy preference relations," Soft Comput., vol. 23, pp. 8057-8073, 2019.

[39] X. Chen, Z.G. Ding, Y.C. Dong, H.M. Liang "Managing consensus with minimum adjustments in group decision making with opinions evolution," IEEE Trans. Syst. Man Cybern. -Syst. DOI: 10.1109/TSMC.2019.2912231.

[40] Y.Z. Wu, Y.C. Dong, J.D. Qin, W. Pedrycz, "Flexible linguistic expressions and consensus reaching with accurate constraints in group decision-making," IEEE Trans. Cybern., vol. 50, no. 6, pp. 2488-2501, 2020.

[41] Z. Zhang, X.Y. Kou, Q.X. Dong, "Additive consistency analysis and improvement for hesitant fuzzy preference relations," Expert Syst. Appl., vol. 98, pp. 118-128, 2018.

[42] Z.B. Wu, X.Y. Yang, J.C. Tu, and X. Chen, "Optimal consistency and consensus models for interval additive preference relations: a discrete distribution perspective," J. Oper. Res. Soc., DOI: 10.1080/01605682.2019.1621219.

[43] G.Q. Zhang, Y.C. Dong, and Y.F. Xu, "Linear optimization modeling of consistency issues in group decision making based on fuzzy preference relations," Expert Syst. Appl., vol. 39, pp. 2415-2420, 2012.

[44] X. Chen, H.J. Zhang, Y.C. Dong, "The fusion process with heterogeneous preference structures in group decision making: a survey," Inf. Fusion, vol. 24, pp. 72-83, 2015.

[45] B.W. Zhang, Y.C. Dong, E. Herrera-Viedma, "Group decision making with heterogeneous preference structures: an automatic mechanism to support consensus reaching," Group Decis. Negotiation, vol. 28, pp. 585-617, 2019

[46] H.J. Zhang, S.H. Zhao, G. Kou, C.C. Li, Y.C. Dong, F. Herrera, “An overview on feedback mechanisms with minimum adjustment or cost in consensus reaching in group decision making: Research paradigms and challenges," Inf. Fusion, vol. 60, pp. 65-79, 2020.

[47] W.Y. Yu, Z. Zhang, and Q.Y. Zhong, "Consensus reaching for MAGDM with multigranular hesitant fuzzy linguistic term sets: a minimum adjustment-based approach," Ann. Oper. Res., vol. 300, pp. 443-466, 2021.

[48]Y.J. Xu, K.W. Li, and H.M. Wang, "Distance-based consensus models for fuzzy and multiplicative preference relations," Inf. Sci., vol. 253, pp. 56-73, 2013.

[49] X. Chen, W.J. Xu, H.M. Liang, and Y.C. Dong, "The classifification based consensus in multi-attribute group decision making," J. Oper. Res. Soc., vol. 71, no. 9, pp. 1375-1389, 2020.

[50] Z.B. Wu, S. Huang, and J.P. Xu, "Multi-stage optimization models for individual consistency and group consensus with preference relations," Eur. J. Oper. Res., vol. 275, pp. 182-194, 2019.
[51] D. Cheng, F.X. Cheng, Z.L. Zhou, Y. Wu, "Reaching a minimum adjustment consensus in social network group decision-making," Inf. Fusion, vol. 59, pp. 30-43, 2020.

[52] J. Xiao, X.L. Wang, H.J. Zhang, "Managing personalized individual semantics and consensus in linguistic distribution large-scale group decision making," Inf. Fusion, vol. 53, pp. 20-34, 2020.

[53] H.H. Zhang, G. Kou, Y. Peng, "Soft consensus cost models for group decision making and economic interpretations," Eur. J. Oper. Res., vol. 277, no. 3, pp. 964-980, 2019.

[54] Q. X. Dong, X. Zhou, and L. Martínez, "A hybrid group decision making framework for achieving agreed solutions based on stable opinions," Inf. Sci., vol. 490, pp. 227-243, Jul. 2019.

[55] G. B. Dantzig, Linear Programming and Extensions, New York: Princeton University Press, 1963.

[56] Z. Zhang, Z.L. Li, Y. Gao, "Consensus reaching for group decision making with multi-granular unbalanced linguistic information: A bounded confidence and minimum adjustment-based approach," Inf. Fusion, vol.74, pp. 96-110, 2021.

[57] Z. Zhang, Y. Gao, Z.L. Li, "Consensus reaching for social network group decision making by considering leadership and bounded confidence," Knowl. Based Syst., vol. 204, 106240, 2020.

[58] B. Chandran, B. Golden, and E. Wasil, "Linear programming models for estimating weights in the analytic hierarchy process," Comput. Oper. Res., vol. 32, no. 9, pp. 2235-2254, 2005.

[59] Y.C. Dong, and E. Herrera-Viedma, "Consistency-Driven Automatic Methodology to Set Interval Numerical Scales of 2-Tuple Linguistic Term Sets and Its Use in the Linguistic GDM With Preference Relation," IEEE Trans. Cybern., vol. 45, no. 4, pp. 780-792, 2015.

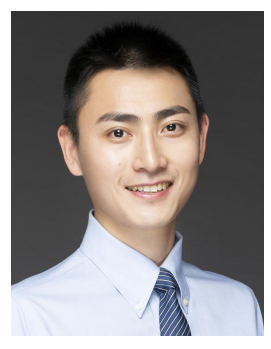

Quanbo Zha received the B.S. and M.S. degrees in vehicle engineering from Southwest Jiaotong University, Chengdu, China, in 2012 and 2015, respectively, and the Ph.D. degree in management science from the Business School, Sichuan University, Chengdu, China, in 2019.

$\mathrm{He}$ is currently a Assistant Professor with the School of Management Science and Real Estate, Chongqing University, Chongqing, China. His research results have been published in refereed journals, including the IEEE Transactions on Systems, Man, And Cybernetics: Systems, IEEE Transactions on Fuzzy Systems, Knowledge-Based Systems, IEEE Transactions on Computational Social Systems, and Financial Innovation, among others. His research interests include opinion dynamics, social network, and decision support systems.

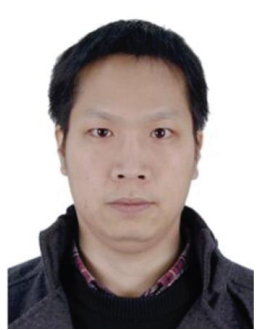

Yucheng Dong received the B.S. and M.S. degrees both in mathematics from Chongqing University, Chongqing, China, in 2002 and 2004, respectively, and the $\mathrm{Ph} . \mathrm{D}$. degree in management from Xi'an Jiaotong University, Xi'an, China, in 2008.

$\mathrm{He}$ is currently a Professor at the Business School, Sichuan University, Chengdu, China. His current research interests include consensus process, computing with words, opinion dynamics, and social network decision making. He has published more than 70 international journal papers in Decision Support Systems, European Journal of Operational Research, 
IEEE Transactions on Big Data, IEEE Transactions on Cybernetics, IEEE Transactions on Fuzzy Systems, IEEE Transactions on Systems, Man, and Cybernetics, Omega, among others.

Dr. Dong is a member of the editorial board of Information Fusion, an Area Editor of Computers \& Industrial Engineering, and an Associate Editor of Group Decision and Negotiation.

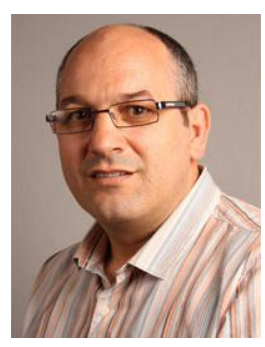

Francisco Chiclana received the B.Sc. and Ph.D. degrees in mathematics from the University of Granada, Granada, Spain, in 1989 and 2000, respectively.

$\mathrm{He}$ is a Professor with the Institute of Artifificial Intelligence and School of Computer Science and Informatics, De Montfort University, Leicester, U.K. Dr. Chiclana is currently a Highly Cited Researcher in Computer Sciences (according to Essential Science Indicators by Clarivate Analytics). $\mathrm{He}$ is an Associate Editor and a Guest Editor for several ISI indexed journals. He has organized and chaired special sessions/workshops in many major international conferences in research areas such as fuzzy preference modeling, decision support systems, consensus, recommender systems, social networks, rationality/consistency, and aggregation.

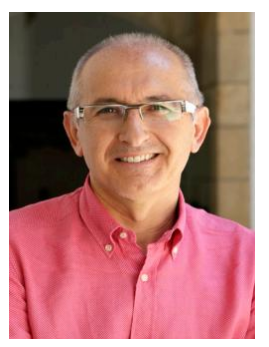

Enrique Herrera-Viedma (Fellow, IEEE) received theM.Sc. and Ph.D. degrees in computer science from the University of Granada, Granada, Spain, in 1993 and 1996, respectively.

He is a Professor of computer science and the Vice-President for Research and Knowledge Transfer with University of Granada, Granada, Spain. His h-index is 82 with more than 24000 citations received in Web of Science and 100 in Google Scholar with more than 40000 cites received. His current research interests include group decision making, consensus models, linguistic modeling, aggregation of information, information retrieval, bibliometric, digital libraries, web quality evaluation, recommender systems, and social media.

Dr. Herrera-Viedma has been identified as one of the world's most influential Researchers by the Shanghai Center and Thomson Reuters/Clarivate Analytics in both computer science and engineering in the years 2014-2020. He is Vice-President for Cybernetics in IEEE SMC and an Associate Editor for several journals such as IEEE Transactions on Fuzzy Systems, IEEE Transactions on Systems, Man, And Cybernetics: Systems, IEEE Transactions on Intelligent Transport System, Information Sciences, Applied Soft Computing, Soft Computing, Fuzzy Optimization and Decision Making, and Knowledge-Based Systems. 